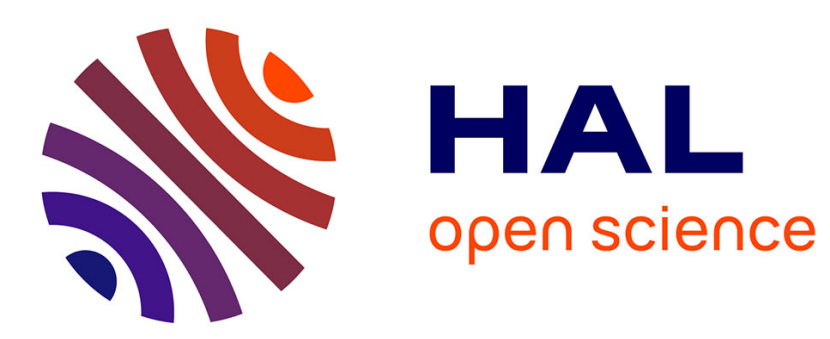

\title{
Mean Reflected Stochastic Differential Equations With Jumps
}

\author{
Philippe Briand, Abir Ghannoum, Céline Labart
}

\section{To cite this version:}

Philippe Briand, Abir Ghannoum, Céline Labart. Mean Reflected Stochastic Differential Equations With Jumps. Advances in Applied Probability, 2020, 52 (2), pp.523-562. hal-01742164v2

HAL Id: hal-01742164

https://hal.univ-smb.fr/hal-01742164v2

Submitted on 19 Dec 2019

HAL is a multi-disciplinary open access archive for the deposit and dissemination of scientific research documents, whether they are published or not. The documents may come from teaching and research institutions in France or abroad, or from public or private research centers.
L'archive ouverte pluridisciplinaire HAL, est destinée au dépôt et à la diffusion de documents scientifiques de niveau recherche, publiés ou non, émanant des établissements d'enseignement et de recherche français ou étrangers, des laboratoires publics ou privés. 


\title{
MEAN REFLECTED STOCHASTIC DIFFERENTIAL EQUATIONS WITH JUMPS
}

\author{
PHILIPPE BRIAND, ABIR GHANNOUM, AND CÉLINE LABART
}

\begin{abstract}
In this paper, a reflected Stochastic Differential Equation with jumps is studied for the case where the constraint acts on the law of the solution rather than on its paths. These reflected SDEs have been approximated in [BCdRGL16] using a numerical scheme based on partical systems, when no jumps occur. The main contribution of this paper is to prove the existence and the uniqueness of the solutions to this kind of reflected SDEs with jumps and to generalize the results obtained in [BCdRGL16] to this context.
\end{abstract}

\section{INTRODUCTION}

Reflected stochastic differential equations have been introduced in the pionneering work of Skorokhod (see [Sko61]), and their numerical approximations by Euler schemes have been widely studied (see [Slo94], [Slo01], [Lep95], [Pet95], [Pet97]). Reflected stochastic differential equations driven by a Lévy process have also been studied in the literature (see [MR85], [KH92]). More recently, reflected backward stochastic differential equations with jumps have been introduced and studied (see [HO03], [EHO05], [HH06], [Ess08], [CM08], [QS14]), as well as their numerical approximation (see [DL16a] and [DL16b]). The main particularity of our work comes from the fact that the constraint acts on the law of the process $X$ rather than on its paths. The study of such equations is linked to the mean field games theory, which has been introduced by Lasry and Lions (see [LL07a], [LL07b], [LL06b], [LL06a]) and whose probabilistic point of view is studied in [CD18a] and [CD18b]. Stochastic differential equations with mean reflection have been introduced by Briand, Elie and $\mathrm{Hu}$ in their backward forms in [BEH18]. In that work, they show that mean reflected stochastic processes exist and are uniquely defined by the associated system of equations of the following form:

$$
\left\{\begin{array}{l}
X_{t}=X_{0}+\int_{0}^{t} b\left(X_{s}\right) d s+\int_{0}^{t} \sigma\left(X_{s}\right) d B_{s}+K_{t}, \quad t \geq 0, \\
\mathbb{E}\left[h\left(X_{t}\right)\right] \geq 0, \quad \int_{0}^{t} \mathbb{E}\left[h\left(X_{s}\right)\right] d K_{s}=0, \quad t \geq 0 .
\end{array}\right.
$$

Due to the fact that the reflection process $K$ depends on the law of the position, the authors of [BCdRGL16], inspired by mean field games, study the convergence of a numerical scheme based on particle systems to compute numerically solutions to (1.1).

In this paper, we extend previous results to the case of jumps, i.e. we study existence and uniqueness of solutions to the following mean reflected stochastic differential equation (MR-SDE in the sequel)

Date: 19th December, 2019. 


$$
\left\{\begin{array}{l}
X_{t}=X_{0}+\int_{0}^{t} b\left(X_{s^{-}}\right) d s+\int_{0}^{t} \sigma\left(X_{s^{-}}\right) d B_{s}+\int_{0}^{t} \int_{E} F\left(X_{s^{-}}, z\right) \tilde{N}(d s, d z)+K_{t}, \quad t \geq 0, \\
\mathbb{E}\left[h\left(X_{t}\right)\right] \geq 0, \quad \int_{0}^{t} \mathbb{E}\left[h\left(X_{s}\right)\right] d K_{s}=0, \quad t \geq 0,
\end{array}\right.
$$

where $E=\mathbb{R}^{*}, \tilde{N}$ is a compensated Poisson measure $\tilde{N}(d s, d z)=N(d s, d z)-\lambda(d z) d s$, and $B$ is a Brownian process independent of $N$. We also propose a numerical scheme based on a particle system to compute numerically solutions to (1.2) and study the rate of convergence of this scheme.

Our main motivation for studying (1.2) comes from financial problems submitted to risk measure constraints. Given any position $X$, its risk measure $\rho(X)$ can be seen as the amount of own fund needed by the investor to hold the position. For example, we can consider the following risk measure: $\rho(X)=\inf \{m: \mathbb{E}[u(m+X)] \geq p\}$ where $u$ is a utility function (concave and increasing) and $p$ is a given threshold (we refer the reader to [ADEH99] and to [FS02] for more details on risk measures). Suppose that we are given a portfolio $X$ of assets whose dynamic, when there is no constraint, follows the jump diffusion model

$$
d X_{t}=b\left(X_{t}\right) d t+\sigma\left(X_{t}\right) d B_{t}+\int_{E} F\left(X_{t-}, z\right) \tilde{N}(d t, d z), \quad t \geq 0 .
$$

Given a risk measure $\rho$, one can ask that $X_{t}$ remains an acceptable position at each time $t$. The constraint rewrites $\mathbb{E}\left[h\left(X_{t}\right)\right] \geq 0$ for $t \geq 0$ where $h=u-p$.

In order to satisfy this constraint, the agent has to add some cash in the portfolio through the time and the dynamic of the wealth of the portfolio becomes

$$
d X_{t}=b\left(X_{t}\right) d t+\sigma\left(X_{t}\right) d B_{t}+\int_{E} F\left(X_{t-}, z\right) \tilde{N}(d t, d z)+d K_{t}, \quad t \geq 0
$$

where $K_{t}$ is the amount of cash added up to time $t$ in the portfolio to balance the "risk" associated to $X_{t}$. Of course, the agent wants to cover the risk in a minimal way, adding cash only when needed: this leads to the Skorokhod condition $\mathbb{E}\left[h\left(X_{t}\right)\right] d K_{t}=0$. Putting together all conditions, we end up with a dynamic of the form (1.2) for the portfolio.

The paper is organized as follows. In Section 2, we show that, under Lipschitz assumptions on $b, \sigma$ and $F$ and bi-Lipchitz assumptions on $h$, the system admits a unique strong solution, i.e. there exists a unique pair of process $(X, K)$ satisfying system (1.2) almost surely, the process $K$ being an increasing and deterministic process. Then, we show that, by adding some regularity on the function $h$, the Stieltjes measure $d K$ is absolutely continuous with respect to the Lebesgue measure and we obtain the explicit expression of its density. In Section 3 we show that the system (1.2) can be seen as the limit of an interacting particle system with oblique reflection of mean field type. This result allows to define in Section 4 an algorithm based on this interacting particle system together with a classical Euler scheme which gives a strong approximation of the solution of (1.2). When $h$ is bi-Lipschitz, this leads to an approximation error in $L^{2}$-sense proportional to $n^{-1}+N^{-\frac{1}{2}}$, where $n$ is the number of points of the discretization grid and $N$ is the number of particles. When $h$ is smooth, we get an approximation error proportional to $n^{-1}+N^{-1}$. By the way, we improve the speed of convergence obtained in [BCdRGL16]. Finally, we illustrate these results numerically in Section 5 . 


\section{Existence, Uniqueness And PROPERTies of the SOlution.}

In this paper, $(\Omega, \mathcal{F}, \mathbb{P})$ is a complete probability space endowed with a standard Brownian motion $B=\left\{B_{t}\right\}_{0 \leq t \leq T}$. $\left\{\mathcal{F}_{t}\right\}_{0 \leq t \leq T}$ is the usual augmented filtration of $B$. Before moving on, we give the following assumptions needed in the sequel.

\section{Assumption (A.1).}

(i) Lipschitz assumption: there exists a constant $C_{p}>0$, such that for all $x, x^{\prime} \in \mathbb{R}$ and $p>0$, we have

$$
\left|b(x)-b\left(x^{\prime}\right)\right|^{p}+\left|\sigma(x)-\sigma\left(x^{\prime}\right)\right|^{p}+\int_{E}\left|F(x, z)-F\left(x^{\prime}, z\right)\right|^{p} \lambda(d z) \leq C_{p}\left|x-x^{\prime}\right|^{p} .
$$

(ii) The random variable $X_{0}$ is square integrable independent of $B_{t}$ and $N_{t}$.

\section{Assumption (A.2).}

(i) The function $h: \mathbb{R} \longrightarrow \mathbb{R}$ is increasing and bi-Lipschitz: there exist $0<m \leq M$ such that

$$
\forall x \in \mathbb{R}, \forall y \in \mathbb{R}, m|x-y| \leq|h(x)-h(y)| \leq M|x-y| .
$$

(ii) The initial condition $X_{0}$ satisfies: $\mathbb{E}\left[h\left(X_{0}\right)\right] \geq 0$.

Assumption (A.3). $\exists p>4$ such that $X_{0} \in \mathrm{L}^{p}$ i.e. $\mathbb{E}\left[\left|X_{0}\right|^{p}\right]<\infty$.

Assumption (A.4). The function h is twice continuously differentiable with bounded derivatives.

2.1. Preliminary results. Consider the function

$$
H \quad: \mathbb{R} \times \mathcal{P}_{1}(\mathbb{R}) \ni(x, \nu) \mapsto \int h(x+z) \nu(d z),
$$

where $\mathcal{P}_{1}(\mathbb{R})$ is the set of probability measures with a finite first-order moment.

Let $\bar{G}_{0}$ be the inverse function in space of $H$ evaluated at 0 :

$$
\bar{G}_{0} \quad: \mathcal{P}_{1}(\mathbb{R}) \ni \nu \mapsto \inf \{x \in \mathbb{R}: H(x, \nu) \geq 0\},
$$

and $G_{0}$ is the positive part of $\bar{G}_{0}$ :

$$
G_{0} \quad: \mathcal{P}_{1}(\mathbb{R}) \ni \nu \mapsto \inf \{x \geq 0: H(x, \nu) \geq 0\} .
$$

We start by studying some properties of $H$ and $G_{0}$.

Lemma 1. Under Assumption (A.2), we have:

(i) For all $\nu$ in $\mathcal{P}_{1}(\mathbb{R})$, the function $H(\cdot, \nu): \mathbb{R} \ni x \mapsto H(x, \nu)$ is bi-Lipschitz:

$$
\forall x, y \in \mathbb{R}, m|x-y| \leq|H(x, \nu)-H(y, \nu)| \leq M|x-y| .
$$

(ii) For all $x$ in $\mathbb{R}$, the function $H(x, \cdot): \mathcal{P}_{1}(\mathbb{R}) \ni \nu \mapsto H(x, \nu)$ satisfies the following Lipschitz inequality:

$$
\forall \nu, \nu^{\prime} \in \mathcal{P}_{1}(\mathbb{R}),\left|H(x, \nu)-H\left(x, \nu^{\prime}\right)\right| \leq\left|\int h(x+\cdot)\left(d \nu-d \nu^{\prime}\right)\right| .
$$

Proof. Lemma 1 ensues from the definition of $H$ (see (2.1)). 
Let $\nu$ and $\nu^{\prime}$ be two probability measures. The Wasserstein- 1 distance between $\nu$ and $\nu^{\prime}$ is defined by:

$$
W_{1}\left(\nu, \nu^{\prime}\right)=\sup _{\varphi 1-\text { Lipschitz }}\left|\int \varphi\left(d \nu-d \nu^{\prime}\right)\right|=\inf _{X \sim \nu ; Y \sim \nu^{\prime}} \mathbb{E}[|X-Y|] .
$$

Thus

$$
\forall \nu, \nu^{\prime} \in \mathcal{P}_{1}(\mathbb{R}),\left|H(x, \nu)-H\left(x, \nu^{\prime}\right)\right| \leq M W_{1}\left(\nu, \nu^{\prime}\right) .
$$

According to Monge-Kantorovitch Theorem, the assertion (2.5) implies that for all $x$ in $\mathbb{R}$, the function $H(x, \cdot)$ is Lipschitz continuous w.r.t. the Wasserstein-1 distance. Then, the regularity of $G_{0}$ is given in the following Lemma:

Lemma 2. Under Assumption (A.2), the function $G_{0}: \mathcal{P}_{1}(\mathbb{R}) \ni \nu \mapsto G_{0}(\nu)$ is Lipschitz continuous:

$$
\left|G_{0}(\nu)-G_{0}\left(\nu^{\prime}\right)\right| \leq \frac{1}{m}\left|\int h\left(\bar{G}_{0}(\nu)+\cdot\right)\left(d \nu-d \nu^{\prime}\right)\right|,
$$

where $\bar{G}_{0}(\nu)$ is the inverse of $H(\cdot, \nu)$ at point 0 . Especially,

$$
\left|G_{0}(\nu)-G_{0}\left(\nu^{\prime}\right)\right| \leq \frac{M}{m} W_{1}\left(\nu, \nu^{\prime}\right) .
$$

Proof. The proof is given in ([BCdRGL16], Lemma 2.5).

2.2. Existence and uniqueness of the solution of (1.2). The set of Assumptions (A.1)-(A.4) will be used as follows:

- The existence and uniqueness results are stated under the standard assumption for SDEs (A.1) and the assumption used in [BEH18] (A.2).

- The convergence of particle systems is proved under (A.3).

- Some of the results will be improved under the smoothness assumption (A.4).

Firstly, we recall the existence and uniqueness result of [BEH18] in the case of SDEs.

Definition 1. A couple of processes $(X, K)$ is said to be a flat deterministic solution to (1.2) if $(X, K)$ satisfy (1.2) with $K$ being a non-decreasing continuous deterministic function with $K_{0}=0$.

Given this definition we have the following result.

Theorem 1. Under Assumptions (A.1) and (A.2), the mean reflected SDE (1.2) has a unique deterministic flat solution $(X, K)$. Moreover,

$$
\forall t \geq 0, K_{t}=\sup _{s \leq t} \inf \left\{x \geq 0: \mathbb{E}\left[h\left(x+U_{s}\right)\right] \geq 0\right\}=\sup _{s \leq t} G_{0}\left(\mu_{s}\right),
$$

where $\left(U_{t}\right)_{0 \leq t \leq T}$ is the process defined by:

$$
U_{t}=X_{0}+\int_{0}^{t} b\left(X_{s^{-}}\right) d s+\int_{0}^{t} \sigma\left(X_{s^{-}}\right) d B_{s}+\int_{0}^{t} \int_{E} F\left(X_{s^{-}}, z\right) \tilde{N}(d s, d z)
$$

and $\left(\mu_{t}\right)_{0 \leq t \leq T}$ is the family of marginal laws of $\left(U_{t}\right)_{0 \leq t \leq T}$.

Proof. We refer to [BEH18], for the proof in the case of continuous backward SDEs. We present here the proof of the forward case with jumps.

Let us consider the set $\mathcal{C}^{2}=\left\{X \mathcal{F}\right.$-adapted càdlàg, $\left.\mathbb{E}\left(\sup _{t \leq T}\left|X_{t}\right|^{2}\right)<\infty\right\}$, and let $\hat{X} \in \mathcal{C}^{2}$ be a given process. We define

$$
\hat{U}_{t}=X_{0}+\int_{0}^{t} b\left(\hat{X}_{s^{-}}\right) d s+\int_{0}^{t} \sigma\left(\hat{X}_{s^{-}}\right) d B_{s}+\int_{0}^{t} \int_{E} F\left(\hat{X}_{s^{-}}, z\right) \tilde{N}(d s, d z)
$$


and the function $K$

$$
K_{t}=\sup _{s \leq t} \inf \left\{x \geq 0: \mathbb{E}\left[h\left(x+\hat{U}_{s}\right)\right] \geq 0\right\}=\sup _{s \leq t} G_{0}\left(\hat{\mu}_{s}\right) .
$$

Let us introduce the process $X$ :

$$
X_{t}=X_{0}+\int_{0}^{t} b\left(\hat{X}_{s^{-}}\right) d s+\int_{0}^{t} \sigma\left(\hat{X}_{s^{-}}\right) d B_{s}+\int_{0}^{t} \int_{E} F\left(\hat{X}_{s^{-}}, z\right) \tilde{N}(d s, d z)+K_{t},
$$

where $K$ is given by (2.11), and check that $(X, K)$ is the solution to (1.2) with $U$ replaced by $\hat{U}$. First, based on the definition of $K$, we have $\mathbb{E}\left[h\left(X_{t}\right)\right] \geq 0, K_{t}=G_{0}\left(\hat{\mu}_{t}\right) d K_{t}-$ a.e. and $G_{0}\left(\hat{\mu}_{t}\right)>0 d K_{t}-a . e$. Then, we obtain

$\int_{0}^{t} \mathbb{E}\left[h\left(X_{s}\right)\right] d K_{s}=\int_{0}^{t} \mathbb{E}\left[h\left(\hat{U}_{s}+K_{s}\right)\right] d K_{s}=\int_{0}^{t} \mathbb{E}\left[h\left(\hat{U}_{s}+G_{0}\left(\hat{\mu}_{s}\right)\right)\right] d K_{s}=\int_{0}^{t} \mathbb{E}\left[h\left(\hat{U}_{s}+G_{0}\left(\hat{\mu}_{s}\right)\right)\right] \mathbf{1}_{G_{0}\left(\hat{\mu}_{s}\right)>0} d K_{s}$.

Moreover, since $h$ is continuous, we have $\mathbb{E}\left[h\left(\hat{U}_{s}+G_{0}\left(\hat{\mu}_{s}\right)\right)\right]=0$ as soon as $G_{0}\left(\hat{\mu}_{s}\right)>0$, so that

$$
\int_{0}^{t} \mathbb{E}\left[h\left(X_{s}\right)\right] d K_{s}=0 .
$$

Second, choose the map $\Phi: \mathcal{C}^{2} \longrightarrow \mathcal{C}^{2}$ which associates to $\hat{X}$ the process $X$, solution to (1.2). Let us prove that $\Phi$ is a contraction. Using the same Brownian motion and Poisson process, we consider $\hat{X}$ and $\hat{X}^{\prime} \in \mathcal{C}^{2}$ and $K$ and $K^{\prime}$ defined by (2.11). From Assumption (A.1), and by using Cauchy-Schwartz and Doob inequality, we get

$$
\begin{aligned}
\mathbb{E}\left[\sup _{t \leq T}\left|X_{t}-X_{t}^{\prime}\right|^{2}\right] \leq & 4 \mathbb{E}\left[\operatorname { s u p } _ { t \leq T } \left\{\left|\int_{0}^{t}\left(b\left(\hat{X}_{s^{-}}\right)-b\left(\hat{X}_{s^{-}}^{\prime}\right)\right) d s\right|^{2}+\left|\int_{0}^{t}\left(\sigma\left(\hat{X}_{s^{-}}\right)-\sigma\left(\hat{X}_{s^{-}}^{\prime}\right)\right) d B_{s}\right|^{2}\right.\right. \\
& \left.\left.+\left|\int_{0}^{t} \int_{E}\left(F\left(\hat{X}_{s^{-}}, z\right)-F\left(\hat{X}_{s^{-}}^{\prime}, z\right)\right) \tilde{N}(d s, d z)\right|^{2}+\left|K_{t}-K_{t}^{\prime}\right|^{2}\right\}\right] \\
\leq & 4\left\{\mathbb{E}\left[\sup _{t \leq T} t \int_{0}^{t}\left|b\left(\hat{X}_{s^{-}}\right)-b\left(\hat{X}_{s^{-}}^{\prime}\right)\right|^{2} d s\right]+\mathbb{E}\left[\sup _{t \leq T}\left|\int_{0}^{t}\left(\sigma\left(\hat{X}_{s^{-}}\right)-\sigma\left(\hat{X}_{s^{-}}^{\prime}\right)\right) d B_{s}\right|^{2}\right]\right. \\
& \left.+\mathbb{E}\left[\sup _{t \leq T}\left|\int_{0}^{t} \int_{E}\left(F\left(\hat{X}_{s^{-}}, z\right)-F\left(\hat{X}_{s^{-}}^{\prime}, z\right)\right) \tilde{N}(d s, d z)\right|^{2}\right]+\sup _{t \leq T}\left|K_{t}-K_{t}^{\prime}\right|^{2}\right\} \\
\leq & C\left\{T \mathbb{E}\left[\int_{0}^{T}\left|b\left(\hat{X}_{s^{-}}\right)-b\left(\hat{X}_{s^{-}}^{\prime}\right)\right|^{2} d s\right]+\mathbb{E}\left[\int_{0}^{T}\left|\sigma\left(\hat{X}_{s^{-}}\right)-\sigma\left(\hat{X}_{s^{-}}^{\prime}\right)\right|^{2} d s\right]\right. \\
& \left.+\int_{0}^{T} \int_{E} \mathbb{E}\left[\left|F\left(\hat{X}_{s^{-}}, z\right)-F\left(\hat{X}_{s^{-}}^{\prime}, z\right)\right|^{2}\right] \lambda(d z) d s+\sup _{t \leq T}\left|K_{t}-K_{t}^{\prime}\right|^{2}\right\} \\
\leq & C\left\{T^{2} C_{1} \mathbb{E}\left[\sup _{t \leq T}\left|\hat{X}_{t^{-}}-\hat{X}_{t^{-}}^{\prime}\right|^{2}\right]+T C_{1} \mathbb{E}\left[\sup _{t \leq T}\left|\hat{X}_{t^{-}}-\hat{X}_{t^{-}}^{\prime}\right|^{2}\right]\right. \\
& \left.+T C_{1} \mathbb{E}\left[\sup _{t \leq T}\left|\hat{X}_{t^{-}}-\hat{X}_{t^{-}}^{\prime}\right|^{2}\right]+\sup _{t \leq T}\left|K_{t}-K_{t}^{\prime}\right|^{2}\right\} \\
\leq & C\left(T^{2} C_{1}+T C_{2}\right) \mathbb{E}\left[\sup _{t \leq T}\left|\hat{X}_{t}-\hat{X}_{t}^{\prime}\right|^{2}\right]+C \sup _{t \leq T}\left|K_{t}-K_{t}^{\prime}\right|^{2} .
\end{aligned}
$$

From the representation (2.11) of the process $K$ and Lemma 2, we have that 


$$
\begin{aligned}
\sup _{t \leq T}\left|K_{t}-K_{t}^{\prime}\right|^{2} & \leq \frac{M}{m} \mathbb{E}\left[\sup _{t \leq T}\left|\hat{U}_{t}-\hat{U}_{t}^{\prime}\right|^{2}\right] \\
& \leq C\left(T^{2} C_{1}+T C_{2}\right) \mathbb{E}\left[\sup _{t \leq T}\left|\hat{X}_{t}-\hat{X}_{t}^{\prime}\right|^{2}\right] .
\end{aligned}
$$

This leads to

$$
\mathbb{E}\left[\sup _{t \leq T}\left|X_{t}-X_{t}^{\prime}\right|^{2}\right] \leq C(1+T) T \mathbb{E}\left[\sup _{t \leq T}\left|\hat{X}_{t}-\hat{X}_{t}^{\prime}\right|^{2}\right]
$$

Therefore, there exists a positive $\mathcal{T}$, depending on $b, \sigma, F$ and $h$ only, such that for all $T<\mathcal{T}$, the map $\Phi$ is a contraction. Consequently, we get the existence and uniqueness of solution on $[0, \mathcal{T}]$ and by iterating the construction the result is extended on $\mathbb{R}^{+}$.

\subsection{Regularity results on $K, X$ and $U$.}

Remark 1. In view of this construction, we derive that for all $0 \leq s<t$ :

$$
\begin{aligned}
& K_{t}-K_{s} \\
& =\sup _{s \leq r \leq t} \inf \left\{x \geq 0: \mathbb{E}\left[h\left(x+X_{s}+\int_{s}^{r} b\left(X_{u^{-}}\right) d u+\int_{s}^{r} \sigma\left(X_{u^{-}}\right) d B_{u}+\int_{s}^{r} \int_{E} F\left(X_{u^{-}}, z\right) \tilde{N}(d u, d z)\right)\right] \geq 0\right\} .
\end{aligned}
$$

Proof. From the representation (2.9) of the process $K$, we have

$$
\begin{aligned}
K_{t} & =\sup _{r \leq t} G_{0}\left(U_{r}\right)=\max \left\{\sup _{r \leq s} G_{0}\left(U_{r}\right), \sup _{s \leq r \leq t} G_{0}\left(U_{r}\right)\right\} \\
& =\max \left\{K_{s}, \sup _{s \leq r \leq t} G_{0}\left(U_{r}\right)\right\} \\
& =\max \left\{K_{s}, \sup _{s \leq r \leq t} G_{0}\left(X_{s}-K_{s}+U_{r}-U_{s}\right)\right\} \\
& =\max \left\{K_{s}, \sup _{s \leq r \leq t}\left[\bar{G}_{0}\left(X_{s}-K_{s}+U_{r}-U_{s}\right)^{+}\right]\right\} .
\end{aligned}
$$

By the definition of $\bar{G}_{0}$, we observe that for all $y \in \mathbb{R}, \bar{G}_{0}(X+y)=\bar{G}_{0}(X)-y$, so we get

$$
\begin{aligned}
K_{t} & =\max \left\{K_{s}, \sup _{s \leq r \leq t}\left[\left(K_{s}+\bar{G}_{0}\left(X_{s}+U_{r}-U_{s}\right)\right)^{+}\right]\right\} \\
& =K_{s}+\max \left\{0, \sup _{s \leq r \leq t}\left[\left(K_{s}+\bar{G}_{0}\left(X_{s}+U_{r}-U_{s}\right)\right)^{+}-K_{s}\right]\right\} .
\end{aligned}
$$

Note that $\sup _{r}\left(f(r)^{+}\right)=\left(\sup _{r} f(r)\right)^{+}=\max \left(0, \sup _{r} f(r)\right)$ for all function $f$, and obviously

$$
\begin{aligned}
K_{t} & =K_{s}+\sup _{s \leq r \leq t}\left[\left\{\left(K_{s}+\bar{G}_{0}\left(X_{s}+U_{r}-U_{s}\right)\right)^{+}-K_{s}\right\}^{+}\right] \\
& =K_{s}+\sup _{s \leq r \leq t}\left[\left(\bar{G}_{0}\left(X_{s}+U_{r}-U_{s}\right)\right)^{+}\right] \\
& =K_{s}+\sup _{s \leq r \leq t} G_{0}\left(X_{s}+U_{r}-U_{s}\right),
\end{aligned}
$$

and so

$$
K_{t}-K_{s}=\sup _{s \leq r \leq t} G_{0}\left(X_{s}+U_{r}-U_{s}\right) .
$$


Proposition 1. Suppose that Assumptions (A.1) and (A.2) hold. Then, for all $p \geq 2$, there exists a positive constant $K_{p}$, depending on $T, b, \sigma, F$ and $h$ such that

(i) $\mathbb{E}\left[\sup _{t \leq T}\left|X_{t}\right|^{p}\right] \leq K_{p}\left(1+\mathbb{E}\left[\left|X_{0}\right|^{p}\right]\right)$.

(ii) $\forall 0 \leq s \leq t \leq T, \quad \mathbb{E}\left[\sup _{s \leq u \leq t}\left|X_{u}\right|^{p} \mid \mathcal{F}_{s}\right] \leq C\left(1+\left|X_{s}\right|^{p}\right)$.

Remark 2. Under the same conditions, we conclude that

$$
\mathbb{E}\left[\sup _{t \leq T}\left|U_{t}\right|^{p}\right] \leq K_{p}\left(1+\mathbb{E}\left[\left|X_{0}\right|^{p}\right]\right) .
$$

Proof of (i). We have

$$
\begin{aligned}
\mathbb{E}\left[\sup _{t \leq T}\left|X_{t}\right|^{p}\right] \leq & 5^{p-1}\left\{\mathbb{E}\left|X_{0}\right|^{p}+\mathbb{E} \sup _{t \leq T}\left(\int_{0}^{t}\left|b\left(X_{s^{-}}\right)\right| d s\right)^{p}+\mathbb{E} \sup _{t \leq T}\left|\int_{0}^{t} \sigma\left(X_{s^{-}}\right) d B_{s}\right|^{p}\right. \\
& \left.+\mathbb{E} \sup _{t \leq T}\left|\int_{0}^{t} \int_{E} F\left(X_{s^{-}}, z\right) \tilde{N}(d s, d z)\right|^{p}+K_{T}^{p}\right\} .
\end{aligned}
$$

The last term $K_{T}=\sup _{t \leq T} G_{0}\left(\mu_{t}\right)$ is firstly studied. By using the Lipschitz property of Lemma 2 of $G_{0}$ and the definition of the Wasserstein metric, we have

$$
\forall t \geq 0,\left|G_{0}\left(\mu_{t}\right)\right| \leq \frac{M}{m} \mathbb{E}\left[\left|U_{t}-U_{0}\right|\right],
$$

since $G_{0}\left(\mu_{0}\right)=0$ as $\mathbb{E}\left[h\left(X_{0}\right)\right] \geq 0$ and where $U$ is defined by (4.3). Therefore

$$
\begin{aligned}
\left|K_{T}\right|^{p}=\left|\sup _{t \leq T} G_{0}\left(\mu_{t}\right)\right|^{p} \leq & 3^{p-1}\left(\frac{M}{m}\right)^{p}\left\{\mathbb{E} \sup _{t \leq T}\left(\int_{0}^{t}\left|b\left(X_{s^{-}}\right)\right| d s\right)^{p}+\mathbb{E} \sup _{t \leq T}\left|\int_{0}^{t} \sigma\left(X_{s^{-}}\right) d B_{s}\right|^{p}\right. \\
& \left.+\mathbb{E} \sup _{t \leq T}\left|\int_{0}^{t} \int_{E} F\left(X_{s^{-}}, z\right) \tilde{N}(d s, d z)\right|^{p}\right\}
\end{aligned}
$$

and so

$$
\begin{aligned}
\mathbb{E}\left[\sup _{t \leq T}\left|X_{t}\right|^{p}\right] \leq & C(p, M, m) \mathbb{E}\left[\left|X_{0}\right|^{p}+\sup _{t \leq T}\left(\int_{0}^{t}\left|b\left(X_{s^{-}}\right)\right| d s\right)^{p}+\sup _{t \leq T}\left|\int_{0}^{t} \sigma\left(X_{s^{-}}\right) d B_{s}\right|^{p}\right. \\
& \left.+\sup _{t \leq T}\left|\int_{0}^{t} \int_{E} F\left(X_{s^{-}}, z\right) \tilde{N}(d s, d z)\right|^{p}\right] .
\end{aligned}
$$

Hence, using Assumption (A.1), Cauchy-Schwartz, Doob and BDG inequalities yields

$$
\begin{aligned}
\mathbb{E}\left[\sup _{t \leq T}\left|X_{t}\right|^{p}\right] \leq & C\left\{\mathbb{E}\left[\left|X_{0}\right|^{p}\right]+T^{p-1} \mathbb{E}\left[\int_{0}^{T}\left(1+\left|X_{s^{-}}\right|\right)^{p} d s\right]+C_{1} \mathbb{E}\left[\int_{0}^{T}\left(1+\left|X_{s^{-}}\right|\right)^{2} d s\right]^{\frac{p}{2}}\right. \\
& \left.+C_{2} \mathbb{E}\left[\int_{0}^{T}\left(1+\left|X_{s^{-}}\right|\right)^{p} d s\right]\right\} \\
\leq & C_{1}\left(1+\mathbb{E}\left|X_{0}\right|^{p}\right)+C_{2} \int_{0}^{T} \mathbb{E}\left[\sup _{t \leq r}\left|X_{t}\right|^{p}\right] d r,
\end{aligned}
$$

and from Gronwall's Lemma, we can conclude that for all $p \geq 2$, there exists a positive constant $K_{p}$, depending on $T, b, \sigma, F$ and $h$ such that

$$
\mathbb{E}\left[\sup _{t \leq T}\left|X_{t}\right|^{p}\right] \leq K_{p}\left(1+\mathbb{E}\left[\left|X_{0}\right|^{p}\right]\right)
$$


Proof of (ii). For the first part, we have

$$
\begin{aligned}
X_{u}= & U_{u}+K_{u} \\
= & X_{s}+\left(U_{u}-U_{s}\right)+\left(K_{u}-K_{s}\right) \\
= & X_{s}+\int_{s}^{u} b\left(X_{r^{-}}\right) d r+\int_{s}^{u} \sigma\left(X_{r^{-}}\right) d B_{r}+\int_{s}^{u} \int_{E} F\left(X_{r^{-}}, z\right) \tilde{N}(d r, d z) \\
& +\left(K_{u}-K_{s}\right) .
\end{aligned}
$$

Let us denote $\mathbb{E}_{s}[\cdot]=\mathbb{E}\left[\cdot \mid \mathcal{F}_{s}\right]$. Then we get

$$
\begin{aligned}
\mathbb{E}_{s}\left[\sup _{s \leq u \leq t}\left|X_{u}\right|^{p}\right] \leq & 5^{p-1}\left\{\mathbb{E}_{s}\left[\left|X_{s}\right|^{p}\right]+\mathbb{E}_{s}\left[\sup _{s \leq u \leq t}\left|\int_{s}^{u} b\left(X_{r^{-}}\right) d r\right|^{p}\right]+\mathbb{E}_{s}\left[\sup _{s \leq u \leq t}\left|\int_{s}^{u} \sigma\left(X_{r^{-}}\right) d B_{r}\right|^{p}\right]\right. \\
& \left.+\mathbb{E}_{s}\left[\sup _{s \leq u \leq t}\left|\int_{s}^{u} \int_{E} F\left(X_{r^{-}}, z\right) \tilde{N}(d r, d z)\right|^{p}\right]+\left|K_{t}-K_{s}\right|^{p}\right\} \\
\leq & C\left\{\left|X_{s}\right|^{p}+T^{p-1} \int_{s}^{t} \mathbb{E}_{s}\left[\left|b\left(X_{r^{-}}\right)\right|^{p}\right] d r+\int_{s}^{t} \mathbb{E}_{s}\left[\left|\sigma\left(X_{r^{-}}\right)\right|^{p}\right] d r\right. \\
& \left.+\int_{s}^{t} \int_{E} \mathbb{E}_{s}\left[\left|F\left(X_{r^{-}}, z\right)\right|^{p}\right] \lambda(d z) d r+2\left|K_{T}\right|^{p}\right\} \\
\leq & C(T)\left\{\left|X_{s}\right|^{p}+C_{1} \int_{s}^{t} \mathbb{E}_{s}\left[1+\left|X_{r^{-}}\right|^{p}\right] d r+2\left|K_{T}\right|^{p}\right\} \\
\leq & C C_{1}\left(1+\left|X_{s}\right|^{p}\right)+C_{2} \int_{s}^{t} \mathbb{E}_{s}\left[\sup _{s \leq u \leq r}\left|X_{u^{-}}\right|^{p}\right] d r .
\end{aligned}
$$

Finally, from Gronwall's Lemma, we deduce that for all $0 \leq s \leq t \leq T$, there exists a constant $C$, depending on $p, T, b, \sigma, F$ and $h$ such that

$$
\mathbb{E}\left[\sup _{s \leq u \leq t}\left|X_{u}\right|^{p} \mid \mathcal{F}_{s}\right] \leq C\left(1+\left|X_{s}\right|^{p}\right) .
$$

Proposition 2. Let $p \geq 2$ and let Assumptions (A.1), (A.2) and (A.3) hold. There exists a constant $C$ depending on $p, T, b, \sigma, F$ and $h$ such that

(i) $\forall 0 \leq s<t \leq T, \quad\left|K_{t}-K_{s}\right| \leq C|t-s|^{(1 / 2)}$.

(ii) $\forall 0 \leq s \leq t \leq T, \quad \mathbb{E}\left[\left|U_{t}-U_{s}\right|^{p}\right] \leq C|t-s|$.

(iii) $\forall 0 \leq r<s<t \leq T, \quad \mathbb{E}\left[\left|U_{s}-U_{r}\right|^{p}\left|U_{t}-U_{s}\right|^{p}\right] \leq C|t-r|^{2}$.

Remark 3. Under the same conditions, we conclude that

$$
\forall 0 \leq s \leq t \leq T, \quad \mathbb{E}\left[\left|X_{t}-X_{s}\right|^{p}\right] \leq C|t-s| .
$$

Proof of (i). Let us recall that, for all process $X$,

$$
\begin{gathered}
\bar{G}_{0}(X)=\inf \{x \in \mathbb{R}: \mathbb{E}[h(x+X)] \geq 0\}, \\
G_{0}(X)=\left(\bar{G}_{0}(X)\right)^{+}=\inf \{x \geq 0: \mathbb{E}[h(x+X)] \geq 0\} .
\end{gathered}
$$

From Remark 1, we have

$$
K_{t}-K_{s}=\sup _{s \leq r \leq t} G_{0}\left(X_{s}+U_{r}-U_{s}\right)
$$


Hence, from the previous representation of $K_{t}-K_{s}$, we deduce the $\frac{1}{2}$-Hölder property of the function $t \longmapsto K_{t}$. Indeed, since by definition $G_{0}\left(X_{s}\right)=0$, if $s<t$, by using Lemma 2,

$$
\begin{aligned}
\left|K_{t}-K_{s}\right| & =\sup _{s \leq r \leq t} G_{0}\left(X_{s}+U_{r}-U_{s}\right) \\
& =\sup _{s \leq r \leq t}\left[G_{0}\left(X_{s}+U_{r}-U_{s}\right)-G_{0}\left(X_{s}\right)\right] \\
& =\frac{M}{m} \sup _{s \leq r \leq t} \mathbb{E}\left[\left|U_{r}-U_{s}\right|\right],
\end{aligned}
$$

and so

$$
\begin{aligned}
\left|K_{t}-K_{s}\right| \leq & C\left\{\mathbb{E}\left[\sup _{s \leq r \leq t}\left|\int_{s}^{r} b\left(X_{u^{-}}\right) d u\right|\right]+\left(\mathbb{E}\left[\sup _{s \leq r \leq t}\left|\int_{s}^{r} \sigma\left(X_{u^{-}}\right) d B_{u}\right|^{2}\right]\right)^{1 / 2}\right. \\
& +\left(\mathbb{E}\left[\sup _{s \leq r \leq t}\left|\int_{s}^{r} \int_{E} F\left(X_{u^{-}}, z\right) \tilde{N}(d u, d z)\right|\right)^{2}\right\} \\
\leq & C\left\{\int_{s}^{t} \mathbb{E}\left[\left|b\left(X_{u^{-}}\right)\right|\right] d u+\left(\mathbb{E}\left[\int_{s}^{t}\left|\sigma\left(X_{u^{-}}\right)\right|^{2} d u\right]\right)^{1 / 2}\right. \\
& +\left(\mathbb{E}\left[\int_{s}^{t} \int_{E}\left|F\left(X_{u^{-}}, z\right)\right|^{2} \lambda(d z) d u \mid\right)^{1 / 2}\right\} \\
\leq & C\left\{|t-s| \mathbb{E}\left[1+\sup _{u \leq T}\left|X_{u}\right|\right]+|t-s|^{1 / 2}\left(\mathbb{E}\left[1+\sup _{u \leq T}\left|X_{u}\right|^{2}\right]\right)^{1 / 2}\right\} .
\end{aligned}
$$

Therefore, if $X_{0} \in \mathrm{L}^{p}$ for some $p \geq 2$, it follows from Proposition 1 that

$$
\left|K_{t}-K_{s}\right| \leq C|t-s|^{1 / 2} \text {. }
$$

Proof of (ii).

$$
\begin{aligned}
\mathbb{E}\left[\left|U_{t}-U_{s}\right|^{p}\right] \leq & 4^{p-1} \mathbb{E}\left[\left(\int_{s}^{t}\left|b\left(X_{r^{-}}\right)\right| d r\right)^{p}+\left|\int_{s}^{t} \sigma\left(X_{r^{-}}\right) d B_{r}\right|^{p}\right. \\
& \left.+\left|\int_{s}^{t} \int_{E} F\left(X_{r^{-}}, z\right) \tilde{N}(d r, d z)\right|^{p}\right] \\
\leq & C \sup _{0 \leq r \leq t} \mathbb{E}\left[\left(\int_{s}^{r}\left|b\left(X_{u^{-}}\right)\right| d u\right)^{p}+\left|\int_{s}^{r} \sigma\left(X_{u^{-}}\right) d B_{u}\right|^{p}\right. \\
& \left.+\left|\int_{s}^{r} \int_{E} F\left(X_{u^{-}}, z\right) \tilde{N}(d u, d z)\right|^{p}\right] \\
\leq & C\left\{|t-s|^{p-1} \mathbb{E}\left[\int_{s}^{t}\left(1+\left|X_{u^{-}}\right|\right)^{p} d u\right]+C_{1} \mathbb{E}\left[\left(\int_{s}^{t}\left(1+\left|X_{u^{-}}\right|\right)^{2} d u\right)^{p / 2}\right]\right. \\
& \left.+C_{2} \mathbb{E}\left[\int_{s}^{t}\left(1+\left|X_{u^{-}}\right|\right)^{p} d u\right]\right\} \\
\leq & C_{1} \mathbb{E}\left[1+\sup _{t \leq T}\left|X_{t}\right|^{p}\right]|t-s|^{p}+C_{2} \mathbb{E}\left[\left(1+\sup _{t \leq T}\left|X_{t}\right|^{2}\right)^{p / 2}\right]|t-s|^{p / 2} \\
& +C_{3} \mathbb{E}\left[1+\sup _{t \leq T}\left|X_{t}\right|^{p}\right]|t-s| .
\end{aligned}
$$


Finally, if $X_{0} \in \mathrm{L}^{p}$ for some $p \geq 2$, we conclude that there exists a constant $C$, depending on $p, T, b, \sigma, F$ and $h$ such that

$$
\forall 0 \leq s \leq t \leq T, \quad \mathbb{E}\left[\left|X_{t}-X_{s}\right|^{p}\right] \leq C|t-s| .
$$

Proof of (iii). Let $0 \leq r<s<t \leq T$, we have

$$
\begin{aligned}
\mathbb{E}\left[\left|U_{s}-U_{r}\right|^{p}\left|U_{t}-U_{s}\right|^{p}\right] \leq & \mathbb{E}\left[\left|U_{s}-U_{r}\right|^{p} \mathbb{E}_{s}\left[\left|U_{t}-U_{s}\right|^{p}\right]\right] \\
\leq & C \mathbb{E}\left[| U _ { s } - U _ { r } | ^ { p } \left\{\mathbb{E}_{s}\left[\left|\int_{s}^{t} b\left(X_{s^{-}}\right) d s\right|^{p}\right]+\mathbb{E}_{s}\left[\left|\int_{s}^{t} \sigma\left(X_{s^{-}}\right) d B_{s}\right|^{p}\right]\right.\right. \\
& \left.\left.+\mathbb{E}_{s}\left[\left|\int_{s}^{t} \int_{E} F\left(X_{s^{-}}, z\right) d \tilde{N}(d s, d z)\right|^{p}\right]\right\}\right]
\end{aligned}
$$

Then, from Burkholder-Davis-Gundy inequality, we get

$$
\begin{aligned}
\mathbb{E}\left[\left|U_{s}-U_{r}\right|^{p}\left|U_{t}-U_{s}\right|^{p}\right] \leq & C \mathbb{E}\left[| U _ { s } - U _ { r } | ^ { p } \left\{\mathbb{E}_{s}\left[\left|\int_{s}^{t} b\left(X_{s^{-}}\right) d s\right|^{p}\right]+\left(\mathbb{E}_{s}\left[\int_{s}^{t}\left|\sigma\left(X_{s^{-}}\right)\right|^{2} d s\right]\right)^{p / 2}\right.\right. \\
& \left.\left.+\mathbb{E}_{s}\left[\int_{s}^{t} \int_{E}\left|F\left(X_{s^{-}}, z\right)\right|^{p} \lambda(d z) d s\right]\right\}\right] \\
\leq & C \mathbb{E}\left[| U _ { s } - U _ { r } | ^ { p } \left\{|t-s|^{p}\left(1+\mathbb{E}_{s}\left[\sup _{s \leq u \leq t}\left|X_{u}\right|^{p}\right]\right)\right.\right. \\
& \left.\left.+|t-s|^{p / 2}\left(1+\mathbb{E}_{s}\left[\sup _{s \leq u \leq t}\left|X_{u}\right|^{2}\right]^{p / 2}\right)+|t-s|\left(1+\mathbb{E}_{s}\left[\sup _{s \leq u \leq t}\left|X_{u}\right|^{p}\right]\right)\right\}\right] \\
\leq & C \mathbb{E}\left[\left|U_{s}-U_{r}\right|^{p}\left\{|t-s|\left(1+\mathbb{E}_{s}\left[\sup _{s \leq u \leq t}\left|X_{u}\right|^{p}\right]\right)\right\}\right],
\end{aligned}
$$

thus, from (i) and Proposition 1, we obtain

$$
\begin{aligned}
\mathbb{E}\left[\left|U_{s}-U_{r}\right|^{p}\left|U_{t}-U_{s}\right|^{p}\right] \leq & C_{1}|t-s| \mathbb{E}\left[\left|U_{s}-U_{r}\right|^{p}\right]+C_{2}|t-s| \mathbb{E}\left[\left|U_{s}-U_{r}\right|^{p}\left|X_{s}\right|^{p}\right] \\
\leq & C_{1}|t-s||s-r|+C_{2}|t-s| \mathbb{E}\left[\left|U_{s}-U_{r}\right|^{p}\left(\left|X_{s}-X_{r}\right|^{p}+\left|X_{r}\right|^{p}\right)\right] \\
\leq & C_{1}|t-r|^{2}+C_{2}|t-s| \mathbb{E}\left[2^{p-1}\left|U_{s}-U_{r}\right|^{p}\left(\left|U_{s}-U_{r}\right|^{p}+\left|K_{s}-K_{r}\right|^{p}\right)\right] \\
& +C_{3}|t-s| \mathbb{E}\left[\left|U_{s}-U_{r}\right|^{p}\left|X_{r}\right|^{p}\right] \\
\leq & C_{1}|t-r|^{2}+C_{2}|t-s| \mathbb{E}\left[\left|U_{s}-U_{r}\right|^{2 p}\right]+C_{3}|t-s||s-r|^{p / 2} \mathbb{E}\left[\left|U_{s}-U_{r}\right|^{p}\right] \\
& +C_{4}|t-s| \mathbb{E}\left[\left|U_{s}-U_{r}\right|^{p}\left|X_{r}\right|^{p}\right] \\
\leq & C_{1}|t-r|^{2}+C_{4}|t-s| \mathbb{E}\left[\left|X_{r}\right|^{p} \mathbb{E}_{r}\left[\left|U_{s}-U_{r}\right|^{p}\right]\right] .
\end{aligned}
$$


Following the proof of (ii), we can also get

$$
\mathbb{E}_{r}\left[\left|U_{s}-U_{r}\right|^{p}\right] \leq C|s-r|\left(1+\mathbb{E}_{r}\left[\sup _{r \leq u \leq s}\left|X_{u}\right|^{p}\right]\right) .
$$

Then

$$
\begin{aligned}
\mathbb{E}\left[\left|U_{s}-U_{r}\right|^{p}\left|U_{t}-U_{s}\right|^{p}\right] & \leq C_{1}|t-r|^{2}+C_{2}|t-s||s-r| \mathbb{E}\left[\left|X_{r}\right|^{p}\left(1+\mathbb{E}_{r}\left[\sup _{r \leq u \leq s}\left|X_{u}\right|^{p}\right]\right)\right] \\
& \leq C_{1}|t-r|^{2}+C_{2}|t-r|^{2} \mathbb{E}\left[\left|X_{r}\right|^{p}\left(1+\sup _{r \leq u \leq s}\left|X_{u}\right|^{p}\right)\right] .
\end{aligned}
$$

Under (A.3), we conclude that

$$
\mathbb{E}\left[\left|U_{s}-U_{r}\right|^{p}\left|U_{t}-U_{s}\right|^{p}\right] \leq C|t-r|^{2}, \quad \forall 0 \leq r<s<t \leq T .
$$

2.4. Density of $K$. Consider $\mathcal{L}$ the linear partial operator of second order described by

$$
\mathcal{L} f(x):=b(x) \frac{\partial}{\partial x} f(x)+\frac{1}{2} \sigma \sigma^{*}(x) \frac{\partial^{2}}{\partial x^{2}} f(x)+\int_{E}\left(f(x+F(x, z))-f(x)-F(x, z) f^{\prime}(x)\right) \lambda(d z),
$$

for any twice continuously differentiable function $f$.

Proposition 3. Assume (A.1), (A.2) and (A.4). Let $(X, K)$ be the unique deterministic flat solution to (1.2). Then the process $K$ is Lipschitz continuous and the Stieljes measure $d K$ has the following density

$$
k: \mathbb{R}^{+} \ni t \longmapsto \frac{\left(\mathbb{E}\left[\mathcal{L} h\left(X_{t^{-}}\right)\right]\right)^{-}}{\mathbb{E}\left[h^{\prime}\left(X_{t^{-}}\right)\right]} \mathbf{1}_{\mathbb{E}\left[h\left(X_{t}\right)\right]=0} .
$$

Let us admit for the moment the following result that will be useful for our proof.

Lemma 3. The functions $t \longmapsto \mathbb{E}\left[h\left(X_{t}\right)\right]$ and $t \longmapsto \mathbb{E}\left[\mathcal{L} h\left(X_{t}\right)\right]$ are continuous.

Lemma 4. If $\varphi$ is a continuous function such that, for some $C \geq 0$ and $p \geq 1$,

$$
\forall x \in \mathbb{R}, \quad|\varphi(x)| \leq C\left(1+|x|^{p}\right),
$$

then the function $t \longmapsto \mathbb{E}\left[\varphi\left(X_{t}\right)\right]$ is continuous.

The proof of Lemma 4 is given in Appendix A.1. We may now proceed to the proof of Proposition 3.

Proof. Firstly, we prove that $K$ is Lipschitz continuous. In order to do it, we first prove that $s \longmapsto$ $\bar{G}_{0}\left(\mu_{s}\right)$ is Lipschitz continuous on $[0, T]$. From the definition of $\bar{G}_{0}$, we have $H\left(\bar{G}_{0}\left(\mu_{t}\right), \mu_{t}\right)=0$ and by using (2.4), if $s<t$, we get

$$
\begin{aligned}
\left|\bar{G}_{0}\left(\mu_{s}\right)-\bar{G}_{0}\left(\mu_{t}\right)\right| & \leq \frac{1}{m}\left|H\left(\bar{G}_{0}\left(\mu_{s}\right), \mu_{t}\right)-H\left(\bar{G}_{0}\left(\mu_{t}\right), \mu_{t}\right)\right| \\
& =\frac{1}{m}\left|H\left(\bar{G}_{0}\left(\mu_{s}\right), \mu_{t}\right)\right| \\
& =\frac{1}{m}\left|\mathbb{E}\left[h\left(\bar{G}_{0}\left(\mu_{s}\right)+U_{t}\right)\right]\right| \\
& =\frac{1}{m}\left|\mathbb{E}\left[h\left(\bar{G}_{0}\left(\mu_{s}\right)+U_{s}+\int_{s}^{t} b\left(X_{r^{-}}\right) d r+\int_{s}^{t} \sigma\left(X_{r^{-}}\right) d B_{r}+\int_{s}^{t} \int_{E} F\left(X_{r^{-}}, z\right) \tilde{N}(d r, d z)\right)\right]\right|
\end{aligned}
$$


From Itô's formula, we obtain

$$
\begin{aligned}
h\left(\bar{G}_{0}\left(\mu_{s}\right)+U_{t}\right)= & h\left(\bar{G}_{0}\left(\mu_{s}\right)+U_{s}\right)+\int_{s}^{t} b\left(X_{r^{-}}\right) h^{\prime}\left(\bar{G}_{0}\left(\mu_{s}\right)+U_{r^{-}}\right) d r+\int_{s}^{t} \sigma\left(X_{r^{-}}\right) h^{\prime}\left(\bar{G}_{0}\left(\mu_{s}\right)+U_{r^{-}}\right) d B_{r} \\
& +\int_{s}^{t} \int_{E} F\left(X_{r^{-}}, z\right) h^{\prime}\left(\bar{G}_{0}\left(\mu_{s}\right)+U_{r^{-}}\right) \tilde{N}(d r, d z)+\frac{1}{2} \int_{s}^{t} \sigma^{2}\left(X_{r^{-}}\right) h^{\prime \prime}\left(\bar{G}_{0}\left(\mu_{s}\right)+U_{r^{-}}\right) d r \\
& +\int_{s}^{t} \int_{E} m(r, z) \lambda(d z) d r+\int_{s}^{t} \int_{E} m(r, z) \tilde{N}(d r, d z),
\end{aligned}
$$

with

$$
m(r, z)=\left(h\left(\bar{G}_{0}\left(\mu_{s}\right)+U_{r^{-}}+F\left(X_{r^{-}}, z\right)\right)-h\left(\bar{G}_{0}\left(\mu_{s}\right)+U_{r^{-}}\right)-F\left(X_{r^{-}}, z\right) h^{\prime}\left(\bar{G}_{0}\left(\mu_{s}\right)+U_{r^{-}}\right)\right) .
$$

This yields

$$
\begin{aligned}
h\left(\bar{G}_{0}\left(\mu_{s}\right)+U_{t}\right)= & h\left(\bar{G}_{0}\left(\mu_{s}\right)+U_{s}\right)+\int_{s}^{t} \overline{\mathcal{L}}_{X_{r^{-}}} h\left(\bar{G}_{0}\left(\mu_{s}\right)+U_{r^{-}}\right) d r+\int_{s}^{t} \sigma\left(X_{r^{-}}\right) h^{\prime}\left(\bar{G}_{0}\left(\mu_{s}\right)+U_{r^{-}}\right) d B_{r} \\
& +\int_{s}^{t} \int_{E}\left(h\left(\bar{G}_{0}\left(\mu_{s}\right)+U_{r^{-}}+F\left(X_{r^{-}}, z\right)\right)-h\left(\bar{G}_{0}\left(\mu_{s}\right)+U_{r^{-}}\right)\right) \tilde{N}(d r, d z),
\end{aligned}
$$

where

$$
\overline{\mathcal{L}}_{y} f(x):=b(y) \frac{\partial}{\partial x} f(x)+\frac{1}{2} \sigma \sigma^{*}(y) \frac{\partial^{2}}{\partial x^{2}} f(x)+\int_{E}\left(f(x+F(y, z))-f(x)-F(y, z) f^{\prime}(x)\right) \lambda(d z) .
$$

Therefore,

$$
\begin{aligned}
\mathbb{E}\left[h\left(\bar{G}_{0}\left(\mu_{s}\right)+U_{t}\right)\right] & =\mathbb{E}\left[h\left(\bar{G}_{0}\left(\mu_{s}\right)+U_{s}\right)\right]+\int_{s}^{t} \mathbb{E}\left[\overline{\mathcal{L}}_{X_{r^{-}}} h\left(\bar{G}_{0}\left(\mu_{s}\right)+U_{r^{-}}\right)\right] d r \\
& =H\left(\bar{G}_{0}\left(\mu_{s}\right), \mu_{s}\right)+\int_{s}^{t} \mathbb{E}\left[\overline{\mathcal{L}}_{X_{r^{-}}} h\left(\bar{G}_{0}\left(\mu_{s}\right)+U_{r^{-}}\right)\right] d r \\
& =\int_{s}^{t} \mathbb{E}\left[\overline{\mathcal{L}}_{X_{r^{-}}} h\left(\bar{G}_{0}\left(\mu_{s}\right)+U_{r^{-}}\right)\right] d r
\end{aligned}
$$

Consequently, the result immediately follows from the fact that $h$ has bounded derivatives and $\sup _{s<T}\left|X_{s}\right|$ is a square integrable random variable for each $T>0$ (see Proposition 1).

Finally, we deduce that $K$ is Lipschitz continuous and so has a bounded density on $[0, T]$ for each $T>0$ (see Proposition 2.7 in [BCdRGL16] for more details).

Secondly, let us find the density of the measure $d K$. For all $0 \leq s \leq t \leq T$, we have

$$
\begin{aligned}
X_{t}= & X_{s}+\int_{s}^{t}\left(b\left(X_{r^{-}}\right)-\int_{E} F\left(X_{r^{-}}, z\right) \lambda(d z)\right) d r+\int_{s}^{t} \sigma\left(X_{r^{-}}\right) d B_{r}+\int_{s}^{t} \int_{E} F\left(X_{r^{-}}, z\right) N(d r, d z) \\
& +K_{t}-K_{s} .
\end{aligned}
$$


Under (A.4) and thanks to Itô's formula we get

$$
\begin{aligned}
h\left(X_{t}\right)-h\left(X_{s}\right)= & \int_{s}^{t} b\left(X_{r^{-}}\right) h^{\prime}\left(X_{r^{-}}\right) d r+\int_{s}^{t} \sigma\left(X_{r^{-}}\right) h^{\prime}\left(X_{r^{-}}\right) d B_{r}+\int_{s}^{t} \int_{E} F\left(X_{r^{-}}, z\right) h^{\prime}\left(X_{r^{-}}\right) \tilde{N}(d r, d z) \\
& +\int_{s}^{t} h^{\prime}\left(X_{r^{-}}\right) d K_{r}+\frac{1}{2} \int_{s}^{t} \sigma^{2}\left(X_{r^{-}}\right) h^{\prime \prime}\left(X_{r^{-}}\right) d r \\
& +\int_{s}^{t} \int_{E}\left(h\left(X_{r^{-}}+F\left(X_{r^{-}}, z\right)\right)-h\left(X_{r^{-}}\right)-F\left(X_{r^{-}}, z\right) h^{\prime}\left(X_{r^{-}}\right)\right) N(d r, d z) \\
= & \int_{s}^{t} b\left(X_{r^{-}}\right) h^{\prime}\left(X_{r^{-}}\right) d r+\int_{s}^{t} \sigma\left(X_{r^{-}}\right) h^{\prime}\left(X_{r^{-}}\right) d B_{r}+\int_{s}^{t} \int_{E} F\left(X_{r^{-}}, z\right) h^{\prime}\left(X_{r^{-}}\right) \tilde{N}(d r, d z) \\
& +\int_{s}^{t} h^{\prime}\left(X_{r^{-}}\right) d K_{r}+\frac{1}{2} \int_{s}^{t} \sigma^{2}\left(X_{r^{-}}\right) h^{\prime \prime}\left(X_{r^{-}}\right) d r \\
& +\int_{s}^{t} \int_{E}\left(h\left(X_{r^{-}}+F\left(X_{r^{-}}\right)\right)-h\left(X_{r^{-}}\right)-F\left(X_{r^{-}}\right) h^{\prime}\left(X_{r^{-}}\right)\right) \lambda(d z) d r \\
& +\int_{s}^{t} \int_{E}\left(h\left(X_{r^{-}}+F\left(X_{r^{-}}, z\right)\right)-h\left(X_{r^{-}}\right)-F\left(X_{r^{-}}, z\right) h^{\prime}\left(X_{r^{-}}\right)\right) \tilde{N}(d r, d z) \\
= & \int_{s}^{t} \mathcal{L} h\left(X_{r^{-}}\right) d r+\int_{s}^{t} h^{\prime}\left(X_{r^{-}}\right) d K_{r}+\int_{s}^{t} \sigma\left(X_{r^{-}}\right) h^{\prime}\left(X_{r^{-}}\right) d B_{r} \\
& +\int_{s}^{t} \int_{E}\left(h\left(X_{r^{-}}+F\left(X_{r^{-}}, z\right)\right)-h\left(X_{r^{-}}\right)\right) \tilde{N}(d r, d z),
\end{aligned}
$$

where $\mathcal{L}$ is given by (2.13). Thus, we obtain

$$
\mathbb{E}\left(\int_{s}^{t} h^{\prime}\left(X_{r^{-}}\right) d K_{r}\right)=\mathbb{E} h\left(X_{t}\right)-\mathbb{E} h\left(X_{s}\right)-\int_{s}^{t} \mathbb{E} \mathcal{L} h\left(X_{r^{-}}\right) d r .
$$

As a conclusion, using (2.15), Lemma 3 and the proof of Proposition 2.7 in [BCdRGL16], we deduce that the measure $d K$ has the following density

$$
k_{t}=\frac{\left(\mathbb{E}\left[\mathcal{L} h\left(X_{t^{-}}\right)\right]\right)^{-}}{\mathbb{E}\left[h^{\prime}\left(X_{t^{-}}\right)\right]} \mathbf{1}_{\mathbb{E}\left[h\left(X_{t}\right)\right]=0}
$$

Proof of Lemma 3. Under Assumption (A.2), and by using Lemma 4, we obtain the continuity of the function $t \longmapsto \mathbb{E} h\left(X_{t}\right)$.

Under the assumptions (A.1), (A.2) and (A.4), we observe that $x \longmapsto \mathcal{L} h\left(X_{t}\right)$ is a continuous function such that, for all $x \in \mathbb{R}$, there exist constants $C_{1}, C_{2}$ and $C_{3}>0$,

and

$$
\begin{gathered}
\left|b(x) h^{\prime}(x)\right| \leq C_{1}(1+|x|), \\
\left|\sigma^{2}(x) h^{\prime \prime}(x)\right| \leq C_{2}\left(1+|x|^{2}\right),
\end{gathered}
$$

$$
\begin{aligned}
\left|\int_{E}\left(h(x+F(x, z))-h(x)-F(x, z) h^{\prime}(x)\right) \lambda(d z)\right| & \leq C_{3} \int_{E}|F(x, z)| \lambda(d z) \\
& \leq C_{3}\left(\int_{E}|F(x, z)-F(0, z)| \lambda(d z)+\int_{E}|F(0, z)| \lambda(d z)\right) \\
& \leq C_{3} \int_{E}|x| \lambda(d z)+C_{3}^{\prime} \\
& \leq C_{3}(1+|x|) .
\end{aligned}
$$

Finally, by using Lemma 4 , we conclude that $t \longmapsto \mathbb{E} \mathcal{L} h\left(X_{t}\right)$ is continuous. 


\section{Approximation of mean Reflected SDEs by an interacting RefleCted particle} SYSTEM.

By using the notations presented in the beginning of Section 2, in particular equation (2.9), the unique solution of the SDE (1.2) can be derived as:

$$
X_{t}=X_{0}+\int_{0}^{t} b\left(X_{s^{-}}\right) d s+\int_{0}^{t} \sigma\left(X_{s^{-}}\right) d B_{s}+\int_{0}^{t} \int_{E} F\left(X_{s^{-}}, z\right) \tilde{N}(d s, d z)+\sup _{s \leq t} G_{0}\left(\mu_{s}\right),
$$

where $\mu_{t}$ stands for the law of

$$
U_{t}=X_{0}+\int_{0}^{t} b\left(X_{s^{-}}\right) d s+\int_{0}^{t} \sigma\left(X_{s^{-}}\right) d B_{s}+\int_{0}^{t} \int_{E} F\left(X_{s^{-}}, z\right) \tilde{N}(d s, d z) .
$$

Let us consider the particle approximation of the above system. In order to do this, let us introduce the particles: for $1 \leq i \leq N$,

$$
X_{t}^{i}=\bar{X}_{0}^{i}+\int_{0}^{t} b\left(X_{s^{-}}^{i}\right) d s+\int_{0}^{t} \sigma\left(X_{s^{-}}^{i}\right) d B_{s}^{i}+\int_{0}^{t} \int_{E} F\left(X_{s^{-}}^{i}, z\right) \tilde{N}^{i}(d s, d z)+\sup _{s \leq t} G_{0}\left(\mu_{s}^{N}\right),
$$

where $\left(B^{i}\right)_{1 \leq i \leq N}$ are independent Brownian motions, $\left(\tilde{N}^{i}\right)_{1 \leq i \leq N}$ are independent compensated Poisson measures, $\left(\bar{X}_{0}^{i}\right)_{1 \leq i \leq N}$ are independent copies of $X_{0}$ and $\mu_{s}^{N}$ represents the empirical distribution at time $s$ of the particles

$$
U_{t}^{i}=\bar{X}_{0}^{i}+\int_{0}^{t} b\left(X_{s^{-}}^{i}\right) d s+\int_{0}^{t} \sigma\left(X_{s^{-}}^{i}\right) d B_{s}^{i}+\int_{0}^{t} \int_{E} F\left(X_{s^{-}}^{i}, z\right) \tilde{N}^{i}(d s, d z), \quad 1 \leq i \leq N,
$$

namely $\mu_{s}^{N}=\frac{1}{N} \sum_{i=1}^{N} \delta_{U_{s}^{i}}$. Note that

$$
\begin{gathered}
G_{0}\left(\mu_{s}^{N}\right)=\inf \left\{x \geq 0: \frac{1}{N} \sum_{i=1}^{N} h\left(x+U_{s}^{i}\right) \geq 0\right\}, \\
K_{t}^{N}=\sup _{s \leq t} G_{0}\left(\mu_{s}^{N}\right) .
\end{gathered}
$$

Now, we can prove the propagation of chaos effect. In order to do it, let us introduce the following independent copies of $X$

$\bar{X}_{t}^{i}=\bar{X}_{0}^{i}+\int_{0}^{t} b\left(\bar{X}_{s^{-}}^{i}\right) d s+\int_{0}^{t} \sigma\left(\bar{X}_{s^{-}}^{i}\right) d B_{s}^{i}+\int_{0}^{t} \int_{E} F\left(\bar{X}_{s^{-}}^{i}, z\right) \tilde{N}^{i}(d s, d z)+\sup _{s \leq t} G_{0}\left(\mu_{s}\right), \quad 1 \leq i \leq N$,

where the Brownian motions and the Poisson processes are the ones used in (3.2).

In addition, we introduce the decoupled particles $\bar{U}^{i}, 1 \leq i \leq N$ :

$$
\bar{U}_{t}^{i}=\bar{X}_{0}^{i}+\int_{0}^{t} b\left(\bar{X}_{s^{-}}^{i}\right) d s+\int_{0}^{t} \sigma\left(\bar{X}_{s^{-}}^{i}\right) d B_{s}^{i}+\int_{0}^{t} \int_{E} F\left(\bar{X}_{s^{-}}^{i}, z\right) \tilde{N}^{i}(d s, d z) .
$$

It is worth noting that the particles $\left(\bar{U}_{t}^{i}\right)_{1 \leq i \leq N}$ are i.i.d.. Furthermore, we introduce $\bar{\mu}^{N}$ as the empirical measure associated to this system of particles.

Remark 4. (i) Under our assumptions, we have $\mathbb{E}\left[h\left(\bar{X}_{0}^{i}\right)\right]=\mathbb{E}\left[h\left(X_{0}\right)\right] \geq 0$. However, there is no reason to have

$$
\frac{1}{N} \sum_{i=1}^{N} h\left(\bar{X}_{0}^{i}\right) \geq 0
$$


even if $N$ is large. As a consequence,

$$
G_{0}\left(\mu_{0}^{N}\right)=\inf \left\{x \geq 0: \frac{1}{N} \sum_{i=1}^{N} h\left(x+\bar{X}_{0}^{i}\right) \geq 0\right\}
$$

is not necessarily equal to 0 . As a byproduct, we have $X_{0}^{i}=\bar{X}_{0}^{i}+G_{0}\left(\mu_{0}^{N}\right)$ and the non decreasing process $\sup _{s \leq t} G_{0}\left(\mu_{s}^{N}\right)$ is not equal to 0 at time $t=0$. Written in this way, the particles defined by (3.2) can not be interpreted as the solution of a reflected SDE. To view the particles as the solution of a reflected SDE, instead of (3.2) one has to solve

$$
\begin{gathered}
X_{t}^{i}=\bar{X}_{0}^{i}+G_{0}\left(\mu_{0}^{N}\right)+\int_{0}^{t} b\left(X_{s^{-}}^{i}\right) d s+\int_{0}^{t} \sigma\left(X_{s^{-}}^{i}\right) d B_{s}^{i}+\int_{0}^{t} \int_{E} F\left(X_{s^{-}}^{i}, z\right) \tilde{N}^{i}(d s, d z)+K_{t}^{N}, \\
\frac{1}{N} \sum_{i=1}^{N} h\left(X_{t}^{i}\right) \geq 0, \quad \int_{0}^{t} \frac{1}{N} \sum_{i=1}^{N} h\left(X_{t}^{i}\right) d K_{s}^{N}=0,
\end{gathered}
$$

with $K^{N}$ non decreasing and $K_{0}^{N}=0$. Since, we do not use this point in the sequel, we will work with the form (3.2).

(ii) Following the proof of Theorem 1, it is easy to demonstrate existence and uniqueness of a solution for the particle approximated system (3.2).

We have the following result concerning the approximation (1.2) by interacting particle system.

Theorem 2. Let $T>0$ and assume that (A.1) and (A.2) hold.

(i) Under Assumption (A.3), there exists a constant $C$ depending on $b, \sigma$ and $F$ such that, for each $j \in\{1, \ldots, N\}$,

$$
\mathbb{E}\left[\sup _{s \leq T}\left|X_{s}^{j}-\bar{X}_{s}^{j}\right|^{2}\right] \leq C \exp \left(C\left(1+\frac{M^{2}}{m^{2}}\right)\left(1+T^{2}\right)\right) \frac{M^{2}}{m^{2}} N^{-1 / 2}
$$

(ii) Under Assumption (A.4), there exists a constant $C$ depending on $b, \sigma$ and $F$ such that, for each $j \in\{1, \ldots, N\}$,

$$
\mathbb{E}\left[\sup _{s \leq T}\left|X_{s}^{j}-\bar{X}_{s}^{j}\right|^{2}\right] \leq C \exp \left(C\left(1+\frac{M^{2}}{m^{2}}\right)\left(1+T^{2}\right)\right) \frac{1+T^{2}}{m^{2}}\left(1+\mathbb{E}\left[\sup _{s \leq T}\left|X_{T}\right|^{2}\right]\right) N^{-1} .
$$

Proof. Let $t>0$. We have, for $r \leq t$,

$$
\begin{aligned}
\left|X_{r}^{j}-\bar{X}_{r}^{j}\right| \leq \mid & \left|\int_{0}^{r} b\left(X_{s^{-}}^{j}\right)-b\left(\bar{X}_{s^{-}}^{j}\right) d s\right|+\left|\int_{0}^{r}\left(\sigma\left(X_{s^{-}}^{j}\right)-\sigma\left(\bar{X}_{s^{-}}^{j}\right)\right) d B_{s}^{i}\right| \\
& +\left|\int_{0}^{r} \int_{E}\left(F\left(X_{s^{-}}^{j}, z\right)-F\left(\bar{X}_{s^{-}}^{j}, z\right)\right) \tilde{N}^{j}(d s, d z)\right|+\left|\sup _{s \leq r} G_{0}\left(\mu_{s}^{N}\right)-\sup _{s \leq r} G_{0}\left(\mu_{s}\right)\right| .
\end{aligned}
$$

Due to the following inequality

$$
\begin{aligned}
\left|\sup _{s \leq r} G_{0}\left(\mu_{s}^{N}\right)-\sup _{s \leq r} G_{0}\left(\mu_{s}\right)\right| & \leq \sup _{s \leq r}\left|G_{0}\left(\mu_{s}^{N}\right)-G_{0}\left(\mu_{s}\right)\right| \leq \sup _{s \leq t}\left|G_{0}\left(\mu_{s}^{N}\right)-G_{0}\left(\mu_{s}\right)\right| \\
& \leq \sup _{s \leq t}\left|G_{0}\left(\mu_{s}^{N}\right)-G_{0}\left(\bar{\mu}_{s}^{N}\right)\right|+\sup _{s \leq t}\left|G_{0}\left(\bar{\mu}_{s}^{N}\right)-G_{0}\left(\mu_{s}\right)\right|,
\end{aligned}
$$

we obtain

$$
\sup _{r \leq t}\left|X_{r}^{j}-\bar{X}_{r}^{j}\right| \leq I_{1}+\sup _{s \leq t}\left|G_{0}\left(\mu_{s}^{N}\right)-G_{0}\left(\bar{\mu}_{s}^{N}\right)\right|+\sup _{s \leq t}\left|G_{0}\left(\bar{\mu}_{s}^{N}\right)-G_{0}\left(\mu_{s}\right)\right|,
$$


where $I_{1}$ is defined by

$$
\begin{aligned}
I_{1}= & \int_{0}^{t}\left|b\left(X_{s^{-}}^{j}\right)-b\left(\bar{X}_{s^{-}}^{j}\right)\right| d s+\sup _{r \leq t}\left|\int_{0}^{r}\left(\sigma\left(X_{s^{-}}^{j}\right)-\sigma\left(\bar{X}_{s^{-}}^{j}\right)\right) d B_{s}^{i}\right| \\
& +\sup _{r \leq t}\left|\int_{0}^{r} \int_{E}\left(F\left(X_{s^{-}}^{j}, z\right)-F\left(\bar{X}_{s^{-}}^{j}, z\right)\right) \tilde{N}^{j}(d s, d z)\right| .
\end{aligned}
$$

Firstly, due to Assumption (A.1), Doob and Cauchy-Schwartz inequalities, we have

$$
\begin{aligned}
\mathbb{E}\left[\left|I_{1}\right|^{2}\right] \leq & C\left\{\mathbb{E}\left[t \int_{0}^{t}\left|b\left(X_{s^{-}}^{j}\right)-b\left(\bar{X}_{s^{-}}^{j}\right)\right|^{2} d s\right]+\mathbb{E}\left[\int_{0}^{t}\left|\sigma\left(X_{s^{-}}^{j}\right)-\sigma\left(\bar{X}_{s^{-}}^{j}\right)\right|^{2} d s\right]\right. \\
& \left.+\mathbb{E}\left[\int_{0}^{t} \int_{E}\left|F\left(X_{s^{-}}^{j}, z\right)-F\left(\bar{X}_{s^{-}}^{j}, z\right)\right|^{2} \lambda(d z) d s\right]\right\} \\
\leq & C\left\{t C_{1} \int_{0}^{t} \mathbb{E}\left[\left|X_{s}^{j}-\bar{X}_{s}^{j}\right|^{2}\right] d s+C_{1} \int_{0}^{t} \mathbb{E}\left[\left|X_{s}^{j}-\bar{X}_{s}^{j}\right|^{2}\right] d s\right. \\
& \left.+C_{1} \int_{0}^{t} \mathbb{E}\left[\left|X_{s}^{j}-\bar{X}_{s}^{j}\right|^{2}\right] d s\right\} \\
\leq & C(1+t) \int_{0}^{t} \mathbb{E}\left[\left|X_{s}^{j}-\bar{X}_{s}^{j}\right|^{2}\right] d s .
\end{aligned}
$$

where $C$ is a constant that depends only on $b, \sigma$ and $F$. Note that $C$ can change from line to line.

Secondly, in view of Lemma 2,

$$
\sup _{s \leq t}\left|G_{0}\left(\mu_{s}^{N}\right)-G_{0}\left(\bar{\mu}_{s}^{N}\right)\right| \leq \frac{M}{m} \sup _{s \leq t} \frac{1}{N} \sum_{i=1}^{N}\left|U_{s}^{i}-\bar{U}_{s}^{i}\right| \leq \frac{M}{m} \frac{1}{N} \sum_{i=1}^{N} \sup _{s \leq t}\left|U_{s}^{i}-\bar{U}_{s}^{i}\right| .
$$

Moreover, taking into account that the variables are exchangeable, Cauchy-Schwartz inequality implies

$$
\mathbb{E}\left[\sup _{s \leq t}\left|G_{0}\left(\mu_{s}^{N}\right)-G_{0}\left(\bar{\mu}_{s}^{N}\right)\right|^{2}\right] \leq \frac{M^{2}}{m^{2}} \frac{1}{N} \sum_{i=1}^{N} \mathbb{E}\left[\sup _{s \leq t}\left|U_{s}^{i}-\bar{U}_{s}^{i}\right|^{2}\right]=\frac{M^{2}}{m^{2}} \mathbb{E}\left[\sup _{s \leq t}\left|U_{s}^{j}-\bar{U}_{s}^{j}\right|^{2}\right] .
$$

Since

$U_{s}^{j}-\bar{U}_{s}^{j}=\int_{0}^{s}\left(b\left(X_{r^{-}}^{j}\right)-b\left(\bar{X}_{r^{-}}^{j}\right)\right) d r+\int_{0}^{s}\left(\sigma\left(X_{r^{-}}^{j}\right)-\sigma\left(\bar{X}_{r^{-}}^{j}\right)\right) d B_{r}^{j}+\int_{0}^{s} \int_{E}\left(F\left(X_{r^{-}}^{j}, z\right)-F\left(\bar{X}_{r^{-}}^{j}\right), z\right) \tilde{N}^{j}(d r, d z)$

and following the previous computations, we get

$$
\mathbb{E}\left[\sup _{s \leq t}\left|G_{0}\left(\mu_{s}^{N}\right)-G_{0}\left(\bar{\mu}_{s}^{N}\right)\right|^{2}\right] \leq C \frac{M^{2}}{m^{2}}(1+t) \int_{0}^{t} \mathbb{E}\left[\left|X_{s}^{j}-\bar{X}_{s}^{j}\right|^{2}\right] d s .
$$

Consequently, combining the previous estimations with equation (3.3) gives

$$
\begin{aligned}
\mathbb{E}\left[\sup _{r \leq t}\left|X_{r}^{j}-\bar{X}_{r}^{j}\right|^{2}\right] & \leq K \int_{0}^{t} \mathbb{E}\left[\left|X_{s}^{j}-\bar{X}_{s}^{j}\right|^{2}\right] d s+4 \mathbb{E}\left[\sup _{s \leq t}\left|G_{0}\left(\bar{\mu}_{s}^{N}\right)-G_{0}\left(\mu_{s}\right)\right|^{2}\right] \\
& \leq K \int_{0}^{t} \mathbb{E}\left[\sup _{r \leq s}\left|X_{r}^{j}-\bar{X}_{r}^{j}\right|^{2}\right] d s+4 \mathbb{E}\left[\sup _{s \leq t}\left|G_{0}\left(\bar{\mu}_{s}^{N}\right)-G_{0}\left(\mu_{s}\right)\right|^{2}\right],
\end{aligned}
$$

where $K=C(1+t)\left(1+M^{2} / m^{2}\right)$. According to Gronwall's Lemma, we get

$$
\mathbb{E}\left[\sup _{r \leq t}\left|X_{r}^{j}-\bar{X}_{r}^{j}\right|^{2}\right] \leq C e^{K t} \mathbb{E}\left[\sup _{s \leq t}\left|G_{0}\left(\bar{\mu}_{s}^{N}\right)-G_{0}\left(\mu_{s}\right)\right|^{2}\right] .
$$


In view of Lemma 2, we have

$$
\mathbb{E}\left[\sup _{s \leq t}\left|G_{0}\left(\bar{\mu}_{s}^{N}\right)-G_{0}\left(\mu_{s}\right)\right|^{2}\right] \leq \frac{1}{m^{2}} \mathbb{E}\left[\sup _{s \leq t}\left|\int h\left(\bar{G}_{0}\left(\mu_{s}\right)+\cdot\right)\left(d \bar{\mu}_{s}^{N}-d \mu_{s}\right)\right|^{2}\right],
$$

which leads to

$$
\mathbb{E}\left[\sup _{r \leq t}\left|X_{r}^{j}-\bar{X}_{r}^{j}\right|^{2}\right] \leq C e^{K t} \frac{1}{m^{2}} \mathbb{E}\left[\sup _{s \leq t}\left|\int h\left(\bar{G}_{0}\left(\mu_{s}\right)+\cdot\right)\left(d \bar{\mu}_{s}^{N}-d \mu_{s}\right)\right|^{2}\right] .
$$

Proof of (i). Since $h$ is at least a Lipschitz function, the rate of convergence will be given by the convergence of empirical measure of i.i.d. diffusion processes. As we consider a uniform convergence in time, getting the usual rate of convergence is not straightforward. If we only suppose that (A.2) holds, we obtain that:

$$
\frac{1}{m^{2}} \mathbb{E}\left[\sup _{s \leq t}\left|\int h\left(\bar{G}_{0}\left(\mu_{s}\right)+\cdot\right)\left(d \bar{\mu}_{s}^{N}-d \mu_{s}\right)\right|^{2}\right] \leq \frac{M^{2}}{m^{2}} \mathbb{E}\left[\sup _{s \leq t} W_{1}^{2}\left(\bar{\mu}_{s}^{N}, \mu_{s}\right)\right]
$$

According to the additional Assumption (A.3), and in view of ([BCdRGL16], Theorem 3.2, Proof of (i)), we have

$$
\mathbb{E}\left[\sup _{s \leq 1} W_{1}^{2}\left(\bar{\mu}_{s}^{N}, \mu_{s}\right)\right] \leq C N^{-1 / 2}
$$

Proof of (ii). Under Assumption (A.4), we can get rid of the supremum in time by using the sharp estimate

$$
\mathbb{E}\left[\sup _{s \leq t}\left|\int h\left(\bar{G}_{0}\left(\mu_{s}\right)+\cdot\right)\left(d \bar{\mu}_{s}^{N}-d \mu_{s}\right)\right|^{2}\right]
$$

According to Proposition 3, let $\psi$ be the Radon-Nikodym derivative of $\bar{G}_{0}(\mu)$. Since $\left(\bar{U}^{i}\right)_{1 \leq i \leq N}$ are independent copies of $U$, we have

$$
\begin{aligned}
R_{N}(s):=\int h\left(\bar{G}_{0}\left(\mu_{s}\right)+\cdot\right)\left(d \bar{\mu}_{s}^{N}-d \mu_{s}\right) & =\frac{1}{N} \sum_{i=1}^{N} h\left(\bar{G}_{0}\left(\mu_{s}\right)+\bar{U}_{s}^{i}\right)-\mathbb{E}\left[h\left(\bar{G}_{0}\left(\mu_{s}\right)+U_{s}\right)\right] \\
& =\frac{1}{N} \sum_{i=1}^{N}\left\{h\left(\bar{G}_{0}\left(\mu_{s}\right)+\bar{U}_{s}^{i}\right)-\mathbb{E}\left[h\left(\bar{G}_{0}\left(\mu_{s}\right)+\bar{U}_{s}^{i}\right)\right]\right\} \\
& =\frac{1}{N} \sum_{i=1}^{N}\left\{h\left(V_{s}^{i}\right)-\mathbb{E}\left[h\left(V_{s}^{i}\right)\right]\right\},
\end{aligned}
$$


where $V^{i}$ is the semi-martingale $s \longmapsto \bar{G}_{0}\left(\mu_{s}\right)+\bar{U}_{s}^{i}$.

It follows from Itô's formula

$$
\begin{aligned}
h\left(V_{s}^{i}\right)= & h\left(V_{0}^{i}\right)+\int_{0}^{s} h^{\prime}\left(V_{r^{-}}^{i}\right) d \bar{G}_{0}\left(\mu_{r}\right)+\int_{0}^{s} b\left(\bar{X}_{r^{-}}^{i}\right) h^{\prime}\left(V_{r^{-}}^{i}\right) d r+\int_{0}^{s} \sigma\left(\bar{X}_{r^{-}}^{i}\right) h^{\prime}\left(V_{r^{-}}^{i}\right) d B_{r}^{i} \\
& +\int_{0}^{s} \int_{E} F\left(\bar{X}_{r^{-}}^{i}, z\right) h^{\prime}\left(V_{r^{-}}^{i}\right) \tilde{N}^{i}(d r, d z)+\frac{1}{2} \int_{0}^{s} \sigma^{2}\left(\bar{X}_{r^{-}}^{i}\right) h^{\prime \prime}\left(V_{r^{-}}^{i}\right) d r \\
& +\int_{0}^{s} \int_{E}\left(h\left(V_{r^{-}}^{i}+F\left(\bar{X}_{r^{-}}^{i}, z\right)\right)-h\left(V_{r^{-}}^{i}\right)-F\left(\bar{X}_{r^{-}}^{i}, z\right) h^{\prime}\left(V_{r^{-}}^{i}\right)\right) \lambda(d z) d r \\
& +\int_{0}^{s} \int_{E}\left(h\left(V_{r^{-}}^{i}+F\left(\bar{X}_{r^{-}}^{i}, z\right)\right)-h\left(V_{r^{-}}^{i}\right)-F\left(\bar{X}_{r^{-}}^{i}, z\right) h^{\prime}\left(V_{r^{-}}^{i}\right)\right) \tilde{N}^{i}(d r, d z) \\
= & h\left(V_{0}^{i}\right)+\int_{0}^{s} h^{\prime}\left(V_{r^{-}}^{i}\right) \psi_{r} d r+\int_{0}^{s} b\left(\bar{X}_{r^{-}}^{i}\right) h^{\prime}\left(V_{r^{-}}^{i}\right) d r+\frac{1}{2} \int_{0}^{s} \sigma^{2}\left(\bar{X}_{r^{-}}^{i}\right) h^{\prime \prime}\left(V_{r^{-}}^{i}\right) d r \\
& +\int_{0}^{s} \int_{E}\left(h\left(V_{r^{-}}^{i}+F\left(\bar{X}_{r^{-}}^{i}, z\right)\right)-h\left(V_{r^{-}}^{i}\right)-F\left(\bar{X}_{r^{-}}^{i}, z\right) h^{\prime}\left(V_{r^{-}}^{i}\right)\right) \lambda(d z) d r \\
& +\int_{0}^{s} \sigma\left(\bar{X}_{r^{-}}^{i}\right) h^{\prime}\left(V_{r^{-}}^{i}\right) d B_{r}^{i}+\int_{0}^{s} \int_{E}\left(h\left(V_{r^{-}}^{i}+F\left(\bar{X}_{r^{-}}^{i}, z\right)\right)-h\left(V_{r^{-}}^{i}\right)\right) \tilde{N}^{i}(d r, d z) \\
= & h\left(V_{0}^{i}\right)+\int_{0}^{s} h^{\prime}\left(V_{r^{-}}^{i}\right) \psi_{r} d r+\int_{0}^{s} \overline{\mathcal{L}}_{\bar{X}_{r^{-}}^{i}} h\left(V_{r^{-}}^{i}\right) d r+\int_{0}^{s} h^{\prime}\left(V_{r^{-}}^{i}\right) \sigma\left(\bar{X}_{r^{-}}^{i}\right) d B_{r}^{i} \\
& +\int_{0}^{s} \int_{E}\left(h\left(V_{r^{-}}^{i}+F\left(\bar{X}_{r^{-}}^{i}, z\right)\right)-h\left(V_{r^{-}}^{i}\right)\right) \tilde{N}^{i}(d r, d z) \\
= & h\left(V_{0}^{i}\right)+\int_{0}^{s}\left\{h^{\prime}\left(V_{r^{-}}^{i}\right) \psi_{r}+\overline{\mathcal{L}}_{\bar{X}_{r^{-}}^{i}} h\left(V_{r^{-}}^{i}\right)\right\} d r+\int_{0}^{s} h^{\prime}\left(V_{r^{-}}^{i}\right) \sigma\left(\bar{X}_{r^{-}}^{i}\right) d B_{r}^{i} \\
& +\int_{0}^{s} \int_{E}\left(h\left(V_{r^{-}}^{i}+F\left(\bar{X}_{r^{-}}^{i}, z\right)\right)-h\left(V_{r^{-}}^{i}\right)\right) \tilde{N}^{i}(d r, d z),
\end{aligned}
$$

Taking expectation gives

$$
\begin{aligned}
\mathbb{E}\left[h\left(V_{s}^{i}\right)\right] & =\mathbb{E}\left[h\left(V_{0}^{i}\right)\right]+\int_{0}^{s} \mathbb{E}\left[h^{\prime}\left(V_{r^{-}}^{i}\right) \psi_{r}+\overline{\mathcal{L}}_{\bar{X}_{r^{-}}^{i}} h\left(V_{r^{-}}^{i}\right)\right] d r \\
& =H\left(\bar{G}_{0}\left(\mu_{0}\right), \mu_{0}\right)+\int_{0}^{s} \mathbb{E}\left[h^{\prime}\left(V_{r^{-}}^{i}\right) \psi_{r}+\overline{\mathcal{L}}_{\bar{X}_{r^{-}}^{i}} h\left(V_{r^{-}}^{i}\right)\right] d r \\
& =0+\int_{0}^{s} \mathbb{E}\left[h^{\prime}\left(V_{r^{-}}^{i}\right) \psi_{r}+\overline{\mathcal{L}}_{\bar{X}_{r^{-}}^{i}} h\left(V_{r^{-}}^{i}\right)\right] d r
\end{aligned}
$$

Immediately, we deduce that

$$
\begin{aligned}
R_{N}(s) & =\frac{1}{N} \sum_{i=1}^{N} h\left(V_{0}^{i}\right)+\frac{1}{N} \sum_{i=1}^{N} \int_{0}^{s} C^{i}(r) d r+M_{N}(s)+L_{N}(s) \\
& =\frac{1}{N} \sum_{i=1}^{N} h\left(V_{0}^{i}\right)+\int_{0}^{s}\left(\frac{1}{N} \sum_{i=1}^{N} C^{i}(r)\right) d r+M_{N}(s)+L_{N}(s),
\end{aligned}
$$

where

$$
\begin{gathered}
C^{i}(r)=h^{\prime}\left(V_{r^{-}}^{i}\right) \psi_{r}+\overline{\mathcal{L}}_{\bar{X}_{r^{-}}^{i}} h\left(V_{r^{-}}^{i}\right)-\mathbb{E}\left[h^{\prime}\left(V_{r^{-}}^{i}\right) \psi_{r}+\overline{\mathcal{L}}_{\bar{X}_{r^{-}}^{i}} h\left(V_{r^{-}}^{i}\right)\right], \\
M_{N}(s)=\frac{1}{N} \sum_{i=1}^{N} \int_{0}^{s} h^{\prime}\left(V_{r^{-}}^{i}\right) \sigma\left(\bar{X}_{r^{-}}^{i}\right) d B_{r}^{i}, \\
L_{N}(s)=\frac{1}{N} \sum_{i=1}^{N} \int_{0}^{s} \int_{E}\left(h\left(V_{r^{-}}^{i}+F\left(\bar{X}_{r^{-}}^{i}, z\right)\right)-h\left(V_{r^{-}}^{i}\right)\right) \tilde{N}^{i}(d r, d z) .
\end{gathered}
$$


Then,

$$
\begin{aligned}
\sup _{s \leq t}\left|R_{N}(s)\right| & \leq\left|\frac{1}{N} \sum_{i=1}^{N} h\left(V_{0}^{i}\right)\right|+\sup _{s \leq t} \int_{0}^{s}\left|\frac{1}{N} \sum_{i=1}^{N} C^{i}(r)\right| d r+\sup _{s \leq t}\left|M_{N}(s)\right|+\sup _{s \leq t}\left|L_{N}(s)\right| \\
& \leq\left|\frac{1}{N} \sum_{i=1}^{N} h\left(V_{0}^{i}\right)\right|+\int_{0}^{t}\left|\frac{1}{N} \sum_{i=1}^{N} C^{i}(r)\right| d r+\sup _{s \leq t}\left|M_{N}(s)\right|+\sup _{s \leq t}\left|L_{N}(s)\right| .
\end{aligned}
$$

Since $\left(U^{i}\right)_{1 \leq i \leq N}$ and $\left(\bar{X}^{i}\right)_{1 \leq i \leq N}$ are i.i.d and by using Cauchy-Schwartz inequality, we obtain

$$
\begin{aligned}
\mathbb{E}\left[\sup _{s \leq t}\left|R_{N}(s)\right|^{2}\right] \leq & 4\left\{\mathbb{V}\left[\frac{1}{N} \sum_{i=1}^{N} h\left(V_{0}^{i}\right)\right]+\mathbb{E}\left[\left(\int_{0}^{t}\left|\frac{1}{N} \sum_{i=1}^{N} C^{i}(r)\right| d r\right)^{2}\right]\right. \\
& \left.+\mathbb{E}\left[\sup _{s \leq t}\left|M_{N}(s)\right|^{2}\right]+\mathbb{E}\left[\sup _{s \leq t}\left|L_{N}(s)\right|^{2}\right]\right\} \\
\leq & 4\left\{\mathbb{V}\left[\frac{1}{N} \sum_{i=1}^{N} h\left(V_{0}^{i}\right)\right]+t \mathbb{E}\left[\int_{0}^{t}\left|\frac{1}{N} \sum_{i=1}^{N} C^{i}(r)\right|^{2} d r\right]\right. \\
& \left.+\mathbb{E}\left[\sup _{s \leq t}\left|M_{N}(s)\right|^{2}\right]+\mathbb{E}\left[\sup _{s \leq t}\left|L_{N}(s)\right|^{2}\right]\right\} \\
= & 4\left\{\mathbb{V}\left[\frac{1}{N} \sum_{i=1}^{N} h\left(V_{0}^{i}\right)\right]+t \int_{0}^{t} \mathbb{V}\left(\frac{1}{N} \sum_{i=1}^{N} C^{i}(r)\right) d r\right. \\
& \left.+\mathbb{E}\left[\sup _{s \leq t}\left|M_{N}(s)\right|^{2}\right]+\mathbb{E}\left[\sup _{s \leq t}\left|L_{N}(s)\right|^{2}\right]\right\} .
\end{aligned}
$$

Hence, we get

$$
\begin{aligned}
\mathbb{E}\left[\sup _{s \leq t}\left|R_{N}(s)\right|^{2}\right] \leq & \frac{4}{N} \mathbb{V}\left[h\left(V_{0}\right)\right]+\frac{4 t}{N} \int_{0}^{t} \mathbb{V}(C(r)) d r+4 \mathbb{E}\left[\sup _{s \leq t}\left|M_{N}(s)\right|^{2}\right]+4 \mathbb{E}\left[\sup _{s \leq t}\left|L_{N}(s)\right|^{2}\right] \\
= & \frac{4}{N} \mathbb{V}\left[h\left(V_{0}\right)\right]+\frac{4 t}{N} \int_{0}^{t} \mathbb{V}\left(h^{\prime}\left(V_{r^{-}}\right) \psi_{r}+\overline{\mathcal{L}}_{X_{r^{-}}} h\left(V_{r^{-}}\right)\right) d r \\
& +4 \mathbb{E}\left[\sup _{s \leq t}\left|M_{N}(s)\right|^{2}\right]+4 \mathbb{E}\left[\sup _{s \leq t}\left|L_{N}(s)\right|^{2}\right] .
\end{aligned}
$$

Since $M_{N}$ is a martingale with

$$
\left\langle M_{N}\right\rangle_{t}=\frac{1}{N^{2}} \sum_{i=1}^{N} \int_{0}^{t}\left(h^{\prime}\left(V_{r^{-}}^{i}\right) \sigma\left(\bar{X}_{r^{-}}^{i}\right)\right)^{2} d r,
$$

Doob's inequality leads to

$$
\begin{aligned}
\mathbb{E}\left[\sup _{s \leq t}\left|M_{N}(s)\right|^{2}\right] & \leq 4 \mathbb{E}\left[\left|M_{N}(t)\right|^{2}\right] \\
& =\frac{4}{N^{2}} \sum_{i=1}^{N} \int_{0}^{t} \mathbb{E}\left[\left(h^{\prime}\left(V_{r^{-}}^{i}\right) \sigma\left(\bar{X}_{r^{-}}^{i}\right)\right)^{2}\right] d r \\
& =\frac{4}{N} \int_{0}^{t} \mathbb{E}\left[\left(h^{\prime}\left(V_{r^{-}}\right) \sigma\left(X_{r^{-}}\right)\right)^{2}\right] d r
\end{aligned}
$$


Then, using Doob inequality for the martingale $L_{N}$, we obtain

$$
\begin{aligned}
\mathbb{E}\left[\sup _{s \leq t}\left|L_{N}(s)\right|^{2}\right] \leq & 4 \mathbb{E}\left[\left|L_{N}(t)\right|^{2}\right] \\
= & \frac{4}{N^{2}} \sum_{i=1}^{N} \mathbb{E}\left[\left(\int_{0}^{t} \int_{E}\left(h\left(V_{r^{-}}^{i}+F\left(\bar{X}_{r^{-}}^{i}, z\right)\right)-h\left(V_{r^{-}}^{i}\right)\right) \tilde{N}^{i}(d r, d z)\right)^{2}\right] \\
& +\frac{8}{N^{2}} \sum_{1 \leq i<j \leq N} \mathbb{E}\left[\int_{0}^{t} \int_{E}\left(h\left(V_{r^{-}}^{i}+F\left(\bar{X}_{r^{-}}^{i}, z\right)\right)-h\left(V_{r^{-}}^{i}\right)\right) \tilde{N}^{i}(d r, d z)\right. \\
& \left.\int_{0}^{t} \int_{E}\left(h\left(V_{r^{-}}^{j}+F\left(\bar{X}_{r^{-}}^{j}, z\right)\right)-h\left(V_{r^{-}}^{j}\right)\right) \tilde{N}^{j}(d r, d z)\right] \\
= & \frac{4}{N^{2}} \sum_{i=1}^{N} \int_{0}^{t} \int_{E} \mathbb{E}\left[\left(h\left(V_{r^{-}}^{i}+F\left(\bar{X}_{r^{-}}^{i}, z\right)\right)-h\left(V_{r^{-}}^{i}\right)\right)^{2}\right] \lambda(d z) d r \\
= & \frac{4}{N} \int_{0}^{t} \int_{E} \mathbb{E}\left[\left(h\left(V_{r^{-}}^{i}+F\left(\bar{X}_{r^{-}}^{i}, z\right)\right)-h\left(V_{r^{-}}^{i}\right)\right)^{2}\right] \lambda(d z) d r .
\end{aligned}
$$

Finally, using the fact that $h$ has bounded derivatives, $b, \sigma$ and $F$ are Lipschitz, we get

$$
\mathbb{E}\left[\sup _{s \leq t}\left|R_{N}(s)\right|^{2}\right] \leq C\left(1+t^{2}\right)\left(1+\mathbb{E}\left[\sup _{s \leq t}\left|X_{s}\right|^{2}\right]\right) N^{-1} .
$$

This gives the result coming back to (3.4).

\section{Numerical approximation AND its PERFormance For MRSDE.}

In this section, the numerical approximation of the $\operatorname{SDE}(1.2)$ on $[0, T]$ is studied. Let $0=$ $T_{0}<T_{1}<\cdots<T_{n}=T$ be a subdivision of $[0, T]$ and define "_" the mapping $s \mapsto \underline{s}=T_{k}$ if $s \in\left[T_{k}, T_{k+1}\right), k \in\{0, \cdots, n-1\}$. Let us consider the case of regular subdivisions: for a given integer $n, T_{k}=k T / n, k=0, \ldots, n$.

According to the previous section, we have shown that the particle system, for $1 \leq i \leq N$,

$$
X_{t}^{i}=\bar{X}_{0}^{i}+\int_{0}^{t} b\left(X_{s^{-}}^{i}\right) d s+\int_{0}^{t} \sigma\left(X_{s^{-}}^{i}\right) d B_{s}^{i}+\int_{0}^{t} \int_{E} F\left(X_{s^{-}}^{i}, z\right) \tilde{N}^{i}(d s, d z)+\sup _{s \leq t} G_{0}\left(\mu_{s}^{N}\right),
$$

where

$$
\begin{aligned}
\mu_{t}^{N} & =\frac{1}{N} \sum_{i=1}^{N} \delta_{U_{t}^{i}}, \\
U_{t}^{i} & =\bar{X}_{0}^{i}+\int_{0}^{t} b\left(X_{s^{-}}^{i}\right) d s+\int_{0}^{t} \sigma\left(X_{s^{-}}^{i}\right) d B_{s}^{i}+\int_{0}^{t} \int_{E} F\left(X_{s^{-}}^{i}, z\right) \tilde{N}^{i}(d s, d z), \quad 1 \leq i \leq N,
\end{aligned}
$$

$B^{i}$ being independent Brownian motions, $N^{i}$ being independent Poisson processes and $\bar{X}_{0}^{i}$ being independent copies of $X_{0}$, converges to the solution of (1.2). Hence, to determine the numerical approximation, we apply an Euler scheme to this particle system. The discrete version of the particle system is: for $1 \leq i \leq N$,

$$
\tilde{X}_{t}^{i}=\bar{X}_{0}^{i}+\int_{0}^{t} b\left(\tilde{X}_{\underline{s}^{-}}^{i}\right) d s+\int_{0}^{t} \sigma\left(\tilde{X}_{\underline{s}^{-}}^{i}\right) d B_{s}^{i}+\int_{0}^{t} \int_{E} F\left(\tilde{X}_{\underline{s}^{-}}^{i}, z\right) \tilde{N}^{i}(d s, d z)+\sup _{s \leq t} G_{0}\left(\tilde{\mu}_{\underline{s}}^{N}\right),
$$


where

$$
\begin{aligned}
& \tilde{\mu}_{\underline{t}}^{N}=\frac{1}{N} \sum_{i=1}^{N} \delta_{\tilde{U}_{t}^{i}}, \\
& \tilde{U}_{t}^{i}=\bar{X}_{0}^{i}+\int_{0}^{t} b\left(\tilde{X}_{\underline{s}^{-}}^{i}\right) d s+\int_{0}^{t} \sigma\left(\tilde{X}_{\underline{s}^{-}}^{i}\right) d B_{s}^{i}+\int_{0}^{t} \int_{E} F\left(\tilde{X}_{\underline{s}^{-}}^{i}, z\right) \tilde{N}^{i}(d s, d z), \quad 1 \leq i \leq N .
\end{aligned}
$$

4.1. Scheme. In view of the above notations and taking into account the result on the interacting system of mean reflected particles of the MR-SDE of Section 3 and Remark 1, we deduce the following algorithm for the numerical approximation of the MR-SDE.

Remark 5. It should be pointed out that, at each step $k$ of the algorithm, the increment of the reflection process $K$ is approximated by the increment of the following approximation:

$$
\Delta_{k} \hat{K}^{N}:=\sup _{l \leq k} G_{0}\left(\tilde{\mu}_{T_{l}}^{N}\right)-\sup _{l \leq k-1} G_{0}\left(\tilde{\mu}_{T_{l}}^{N}\right) .
$$

First, we consider the special case when the SDE is defined by

$$
\left\{\begin{array}{l}
X_{t}=X_{0}+\int_{0}^{t} b\left(X_{s^{-}}\right) d s+\int_{0}^{t} \sigma\left(X_{s^{-}}\right) d B_{s}+\int_{0}^{t} F\left(X_{s^{-}}\right) d \tilde{N}_{s}+K_{t}, \quad t \geq 0, \\
\mathbb{E}\left[h\left(X_{t}\right)\right] \geq 0, \quad \int_{0}^{t} \mathbb{E}\left[h\left(X_{s}\right)\right] d K_{s}=0, \quad t \geq 0 .
\end{array}\right.
$$

where $N$ is a Poisson process with intensity $\lambda$, and $\tilde{N}_{t}=N_{t}-\lambda t$.

According to Remark 1, the increment (4.1) can be estimated by:

$$
\begin{aligned}
& {\widehat{\Delta_{k} K}}^{N}:= \\
& \begin{aligned}
\inf \left\{x \geq 0: \frac{1}{N} \sum_{i=1}^{N} h(\right. & x+\left(\tilde{X}_{T_{k-1}}^{\tilde{\mu}^{N}}\right)^{i}+\frac{T}{n}\left(b\left(\left(\tilde{X}_{T_{k-1}}^{\tilde{\mu}^{N}}\right)^{i}\right)-\lambda F\left(\left(\tilde{X}_{T_{k-1}}^{\tilde{\mu}^{N}}\right)^{j}\right)+\frac{\sqrt{T}}{\sqrt{n}} \sigma\left(\left(\tilde{X}_{T_{k-1}}^{\tilde{\mu}^{N}}\right)^{i}\right) G^{i}\right. \\
& \left.\left.\quad+F\left(\left(\tilde{X}_{T_{k-1}}^{\tilde{\mu}^{N}}\right)^{i}\right) H^{i}\right) \geq 0\right\},
\end{aligned}
\end{aligned}
$$

where $G^{j} \sim \mathcal{N}(0,1)$ and $H^{j} \sim \mathcal{P}(\lambda(T / n))$ and are i.i.d..

In addition, similar procedures as in the proof of Theorem 1 can be used to verify that the increments of the approximated reflection process are equal to the approximation of the increments:

$$
\forall k \in\{1, \cdots n\}:{\widehat{\Delta_{k} K}}^{N}=\Delta_{k} \hat{K}^{N} .
$$




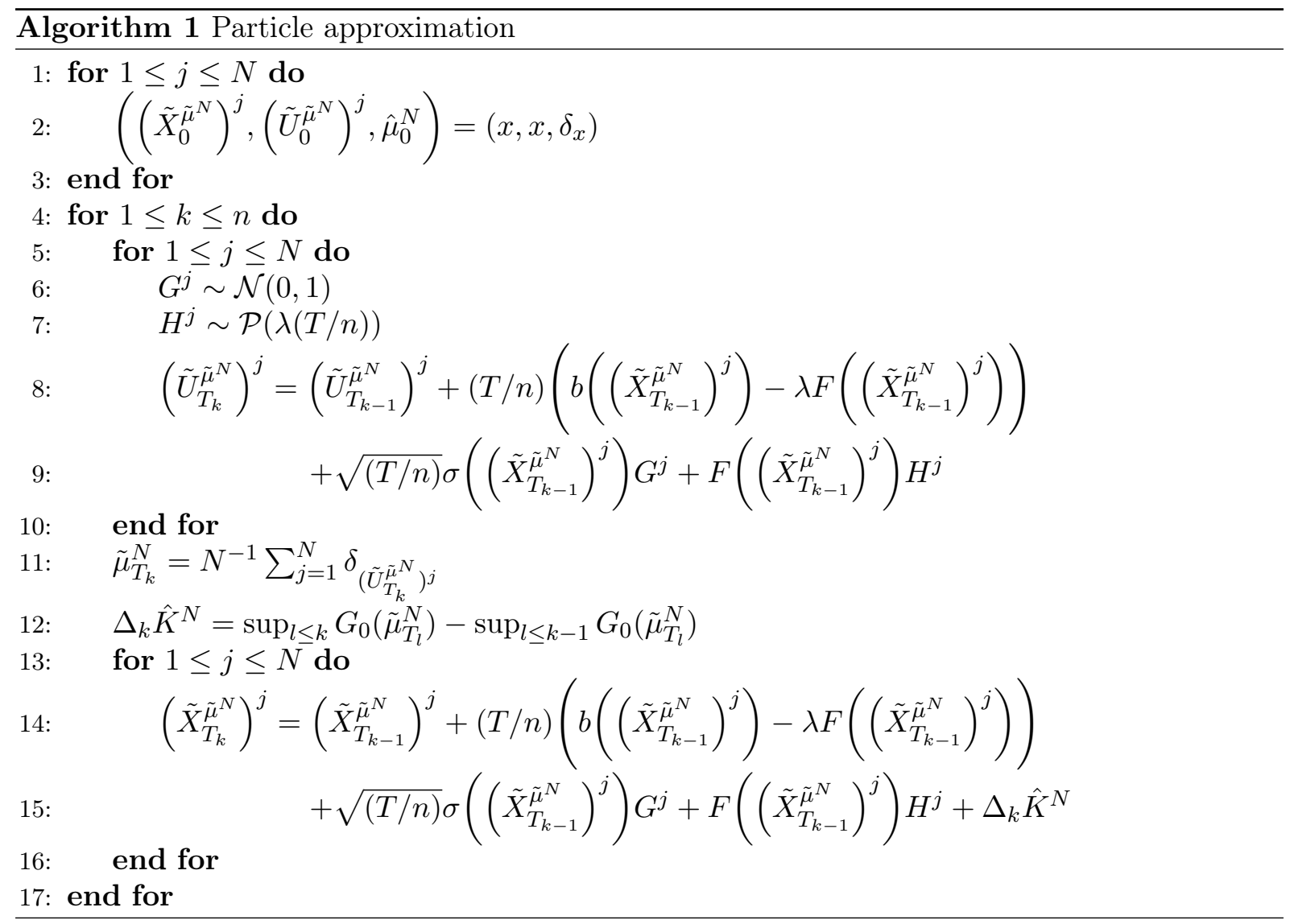

Returning to the general case (1.2), we can see in [YS12], $N=\{N(t):=N(E \times[0, t])\}$ is a stochastic process with intensity $\lambda$ that counts the number of jumps until some given time. The Poisson random measure $N(d z, d t)$ generates a sequence of pairs $\left\{\left(\iota_{i}, \xi_{i}\right), i \in\{1,2, \cdots, N(T)\}\right\}$ for a given finite positive constant $T$ if $\lambda<\infty$. Here $\left\{\iota_{i}, i \in\{1,2, \cdots, N(T)\}\right\}$ is a sequence of increasing nonnegative random variables representing the jump times of a standard Poisson process with intensity $\lambda$, and $\left\{\xi_{i}, i \in\{1,2, \cdots, N(T)\}\right\}$ is a sequence of independent identically distributed random variables, where $\xi_{i}$ is distributed according to $f(z)$, where $\lambda(d z) d t=\lambda f(z) d z d t$. Then, the numerical approximation can equivalently be written in the following form

$$
\begin{aligned}
\bar{X}_{T_{k}}^{j}= & \bar{X}_{T_{k-1}}^{j}+\frac{T}{n}\left(b\left(\bar{X}_{T_{k-1}}^{j}\right)-\int_{E} \lambda F\left(\bar{X}_{T_{k-1}}^{j}, z\right) f(z) d z\right)+\sqrt{\frac{T}{n}} \sigma\left(\bar{X}_{T_{k-1}}^{j}\right) G^{j} \\
& +\sum_{i=H_{T_{k-1}}^{j}+1}^{H_{T_{k}}^{j}} F\left(\bar{X}_{T_{k-1}}^{j}, \xi_{i}\right)+\Delta_{k} \hat{K}^{N},
\end{aligned}
$$

$\Delta_{k} \hat{K}^{N}={\widehat{\Delta_{k} K}}^{N}=$

$\inf \left\{x \geq 0: \frac{1}{N} \sum_{i=1}^{N} h\left(x+\bar{X}_{T_{k-1}}^{j}+\frac{T}{n}\left(b\left(\bar{X}_{T_{k-1}}^{j}\right)-\int_{E} \lambda F\left(\bar{X}_{T_{k-1}}^{j}, z\right) f(z) d z\right)+\sqrt{\frac{T}{n}} \sigma\left(\bar{X}_{T_{k-1}}^{j}\right) G^{j}\right.\right.$

$$
\left.\left.+\sum_{i=H_{T_{k-1}}^{j}+1}^{H_{T_{k}}^{j}} F\left(\bar{X}_{T_{k-1}}^{j}, \xi_{i}\right)\right) \geq 0\right\}
$$

where $G^{j} \sim \mathcal{N}(0,1)$ and $H^{j} \sim \mathcal{P}(\lambda(T / n))$ and are i.i.d.. 


\subsection{Scheme error.}

Proposition 4. (i) Let $T>0, N$ and $n$ be two non-negative integers and let Assumptions (A.1), (A.2) and (A.3) hold. There exists a constant $C$, depending on $T, b, \sigma, F, h$ and $X_{0}$ but independent of $N$, such that: for all $i=1, \ldots, N$

$$
\mathbb{E}\left[\sup _{s \leq t}\left|X_{s}^{i}-\tilde{X}_{s}^{i}\right|^{2}\right] \leq C\left(n^{-1}+N^{-1 / 2}\right) .
$$

(ii) Moreover, if Assumption (A.4) is in force, there exists a constant $C$, depending on $T, b$, $\sigma, F, h$ and $X_{0}$ but independent of $N$, such that: for all $i=1, \ldots, N$

$$
\mathbb{E}\left[\sup _{s \leq t}\left|X_{s}^{i}-\tilde{X}_{s}^{i}\right|^{2}\right] \leq C\left(n^{-1}+N^{-1}\right)
$$

Proof. Let us fix $i \in 1, \ldots, N$ and $T>0$. We have, for $t \leq T$,

$$
\begin{aligned}
\left|X_{s}^{i}-\tilde{X}_{s}^{i}\right| \leq & \left|\int_{0}^{s} b\left(X_{r^{-}}^{i}\right)-b\left(\tilde{X}_{\underline{r}^{-}}^{i}\right) d r\right|+\left|\int_{0}^{s}\left(\sigma\left(X_{r^{-}}^{i}\right)-\sigma\left(\tilde{X}_{\underline{r}^{-}}^{i}\right)\right) d B_{r}^{i}\right| \\
& +\left|\int_{0}^{s} \int_{E}\left(F\left(X_{r^{-}}^{i}, z\right)-F\left(\tilde{X}_{\underline{r}^{-}}^{i}, z\right)\right) \tilde{N}^{i}(d r, d z)\right|+\sup _{r \leq s}\left|G_{0}\left(\mu_{r}^{N}\right)-G_{0}\left(\tilde{\mu}_{\underline{r}}^{N}\right)\right| .
\end{aligned}
$$

Hence, using Assumption (A.1), Cauchy-Schwartz, Doob and BDG inequalities gives

$$
\begin{aligned}
\mathbb{E}\left[\sup _{s \leq t}\left|X_{s}^{i}-\tilde{X}_{s}^{i}\right|^{2}\right] \leq & 4 \mathbb{E}\left[\operatorname { s u p } _ { s \leq t } \left\{\left|\int_{0}^{s}\left(b\left(X_{r^{-}}^{i}\right)-b\left(\tilde{X}_{\underline{r}^{-}}^{i}\right)\right) d r\right|^{2}+\left|\int_{0}^{s}\left(\sigma\left(X_{r^{-}}^{i}\right)-\sigma\left(\tilde{X}_{\underline{r}^{-}}^{i}\right)\right) d B_{r}^{i}\right|^{2}\right.\right. \\
& \left.\left.+\left|\int_{0}^{s} \int_{E}\left(F\left(X_{r^{-}}^{i}, z\right)-F\left(\tilde{X}_{\underline{r}^{-}}^{i}, z\right)\right) \tilde{N}^{i}(d r, d z)\right|^{2}+\sup _{r \leq s}\left|G_{0}\left(\mu_{r}^{N}\right)-G_{0}\left(\tilde{\mu}_{\underline{r}}^{N}\right)\right|^{2}\right\}\right] \\
\leq & C\left\{\mathbb{E}\left[t \int_{0}^{t}\left|b\left(X_{s^{-}}^{i}\right)-b\left(\tilde{X}_{\underline{s}^{-}}^{i}\right)\right|^{2} d s\right]+\mathbb{E}\left[\int_{0}^{t}\left|\sigma\left(X_{s^{-}}^{i}\right)-\sigma\left(\tilde{X}_{\underline{s}^{-}}^{i}\right)\right|^{2} d s\right]\right. \\
& \left.+\mathbb{E}\left[\int_{0}^{t} \int_{E}\left|F\left(X_{s^{-}}^{i}, z\right)-F\left(\tilde{X}_{\underline{s}^{-}}^{i}, z\right)\right|^{2} \lambda(d z) d s\right]+\mathbb{E}\left[\sup _{s \leq t}\left|G_{0}\left(\mu_{s}^{N}\right)-G_{0}\left(\tilde{\mu}_{\underline{s}}^{N}\right)\right|^{2}\right]\right\} \\
\leq & C\left\{T C_{1} \int_{0}^{t} \mathbb{E}\left[\left|X_{s}^{i}-\tilde{X}_{\underline{s}}^{i}\right|^{2}\right] d s+C_{1} \int_{0}^{t} \mathbb{E}\left[\left|X_{s}^{i}-\tilde{X}_{\underline{s}}^{i}\right|^{2}\right] d s\right. \\
& \left.+C_{1} \int_{0}^{t} \mathbb{E}\left[\left|X_{s}^{i}-\tilde{X}_{\underline{s}}^{i}\right|^{2}\right] d s+\mathbb{E}\left[\sup _{s \leq t}\left|G_{0}\left(\mu_{s}^{N}\right)-G_{0}\left(\tilde{\mu}_{\underline{s}}^{N}\right)\right|^{2}\right]\right\} \\
\leq & C \int_{0}^{t} \mathbb{E}\left[\left|X_{s}^{i}-\tilde{X}_{\underline{s}}^{i}\right|^{2}\right] d s+4 \mathbb{E}\left[\sup _{s \leq t}\left|G_{0}\left(\mu_{s}^{N}\right)-G_{0}\left(\tilde{\mu}_{\underline{s}}^{N}\right)\right|^{2}\right] .
\end{aligned}
$$

Denoting by $\left(\mu_{t}^{i}\right)_{0 \leq t \leq T}$ the family of marginal laws of $\left(U_{t}^{i}\right)_{0 \leq t \leq T}$ and $\left(\tilde{\mu}_{\underline{t}}^{i}\right)_{0 \leq t \leq T}$ the family of marginal laws of $\left(\tilde{U}_{t}^{i}\right)_{0 \leq t \leq T}$, we have

$$
\begin{aligned}
\mathbb{E}\left[\sup _{s \leq t}\left|G_{0}\left(\mu_{s}^{N}\right)-G_{0}\left(\tilde{\mu}_{\underline{s}}^{N}\right)\right|^{2}\right] \leq & 3\left\{\mathbb{E}\left[\sup _{s \leq t}\left|G_{0}\left(\mu_{s}^{N}\right)-G_{0}\left(\mu_{s}^{i}\right)\right|^{2}\right]+\sup _{s \leq t}\left|G_{0}\left(\mu_{s}^{i}\right)-G_{0}\left(\tilde{\mu}_{\underline{s}}^{i}\right)\right|^{2}\right. \\
& \left.+\mathbb{E}\left[\sup _{s \leq t}\left|G_{0}\left(\tilde{\mu}_{\underline{s}}^{i}\right)-G_{0}\left(\tilde{\mu}_{\underline{s}}^{N}\right)\right|^{2}\right]\right\}
\end{aligned}
$$


and from Lemma 2 ,

$$
\begin{aligned}
\leq & 3\left\{\frac{1}{m^{2}} \mathbb{E}\left[\sup _{s \leq t}\left|\int h\left(\bar{G}_{0}\left(\mu_{s}^{i}\right)+\cdot\right)\left(d \mu_{s}^{N}-d \mu_{s}^{i}\right)\right|^{2}\right]+\left(\frac{M}{m}\right)^{2} \sup _{s \leq t} W_{1}^{2}\left(\mu_{s}^{i}, \tilde{\mu}_{\underline{s}}^{i}\right)\right. \\
& \left.+\frac{1}{m^{2}} \mathbb{E}\left[\sup _{s \leq t}\left|\int h\left(\bar{G}_{0}\left(\tilde{\mu}_{\underline{s}}^{i}\right)+\cdot\right)\left(d \tilde{\mu}_{\underline{s}}^{N}-d \tilde{\mu}_{\underline{s}}^{i}\right)\right|^{2}\right]\right\} \\
\leq & C\left\{\mathbb{E}\left[\sup _{s \leq t}\left|\int h\left(\bar{G}_{0}\left(\mu_{s}^{i}\right)+\cdot\right)\left(d \mu_{s}^{N}-d \mu_{s}^{i}\right)\right|^{2}\right]+\sup _{s \leq t} \mathbb{E}\left[\left|U_{s}^{i}-\tilde{U}_{\underline{s}}^{i}\right|^{2}\right]\right. \\
& +\mathbb{E}\left[\sup _{s \leq t} \mid \int h\left(\bar{G}_{0}\left(\tilde{\mu}_{\underline{s}}^{i}\right)+\cdot\right)\left(d \tilde{\mu}_{\underline{s}}^{N}-\left.d \tilde{\mu}_{\underline{s}}^{i}\right|^{2}\right]\right\} .
\end{aligned}
$$

Proof of (i). Following the Proof of (i) in Theorem 2, we obtain

$$
\begin{aligned}
& \mathbb{E}\left[\sup _{s \leq t}\left|\int h\left(\bar{G}_{0}\left(\mu_{s}^{i}\right)+\cdot\right)\left(d \mu_{s}^{N}-d \mu_{s}^{i}\right)\right|^{2}\right] \leq C \mathbb{E}\left[\sup _{s \leq t} W_{1}^{2}\left(\mu_{s}^{N}, \mu_{s}^{i}\right)\right] \leq C N^{-1 / 2}, \\
& \mathbb{E}\left[\sup _{s \leq t}\left|\int h\left(\bar{G}_{0}\left(\tilde{\mu}_{\underline{s}}^{i}\right)+\cdot\right)\left(d \tilde{\mu}_{\underline{s}}^{N}-d \tilde{\mu}_{\underline{s}}^{i}\right)\right|^{2}\right] \leq C \mathbb{E}\left[\sup _{s \leq t} W_{1}^{2}\left(\tilde{\mu}_{\underline{s}}^{i}, \tilde{\mu}_{\underline{s}}^{N}\right)\right] \leq C N^{-1 / 2} .
\end{aligned}
$$

From which we can derive the inequality

$$
\begin{aligned}
\mathbb{E}\left[\sup _{s \leq t}\left|G_{0}\left(\mu_{s}^{N}\right)-G_{0}\left(\tilde{\mu}_{\underline{s}}^{N}\right)\right|^{2}\right] & \leq C_{1} \sup _{s \leq t} \mathbb{E}\left[\left|U_{s}^{i}-\tilde{U}_{\underline{s}}^{i}\right|^{2}\right]+C_{2} N^{-1 / 2} \\
& \leq C_{1}\left\{\sup _{s \leq t} \mathbb{E}\left[\left|U_{s}^{i}-\tilde{U}_{s}^{i}\right|^{2}\right]+\sup _{s \leq t} \mathbb{E}\left[\left|\tilde{U}_{s}^{i}-\tilde{U}_{\underline{s}}^{i}\right|^{2}\right]\right\}+C_{2} N^{-1 / 2}
\end{aligned}
$$

For the first term of the right hand side, we can observe that

$$
\begin{aligned}
\left.\sup _{s \leq t} \mathbb{E}\left[\left|U_{s}^{i}-\tilde{U}_{s}^{i}\right|^{2}\right]\right] \leq & \left.\mathbb{E}\left[\sup _{s \leq t}\left|U_{s}^{i}-\tilde{U}_{s}^{i}\right|^{2}\right]\right] \\
\leq & 3 \mathbb{E}\left[\operatorname { s u p } _ { s \leq t } \left\{\left|\int_{0}^{s}\left(b\left(X_{r^{-}}^{i}\right)-b\left(\tilde{X}_{\underline{r}^{-}}^{i}\right)\right) d r\right|^{2}+\left|\int_{0}^{s}\left(\sigma\left(X_{r^{-}}^{i}\right)-\sigma\left(\tilde{X}_{\underline{r}^{-}}^{i}\right)\right) d B_{r}^{i}\right|^{2}\right.\right. \\
& \left.\left.+\left|\int_{0}^{s} \int_{E}\left(F\left(X_{r^{-}}^{i}, z\right)-F\left(\tilde{X}_{\underline{r}^{-}}^{i}, z\right)\right) \tilde{N}^{i}(d r, d z)\right|^{2}\right\}\right] \\
\leq & C\left\{\mathbb{E}\left[t \int_{0}^{t}\left|b\left(X_{s^{-}}^{i}\right)-b\left(\tilde{X}_{\underline{s}^{-}}^{i}\right)\right|^{2} d s\right]+\mathbb{E}\left[\int_{0}^{t}\left|\sigma\left(X_{s^{-}}^{i}\right)-\sigma\left(\tilde{X}_{\underline{s}^{-}}^{i}\right)\right|^{2} d s\right]\right. \\
& \left.+\mathbb{E}\left[\int_{0}^{t} \int_{E}\left|F\left(X_{s^{-}}^{i}, z\right)-F\left(\tilde{X}_{\underline{s}^{-}}^{i}, z\right)\right|^{2} \lambda(d z) d s\right]\right\} \\
\leq & C\left\{T C_{1} \int_{0}^{t} \mathbb{E}\left[\left|X_{s}^{i}-\tilde{X}_{\underline{s}}^{i}\right|^{2}\right] d r+2 C_{1} \int_{0}^{t} \mathbb{E}\left[\left|X_{s}^{i}-\tilde{X}_{\underline{s}}^{i}\right|^{2}\right] d r\right\} \\
\leq & C \int_{0}^{t} \mathbb{E}\left[\left|X_{s}^{i}-\tilde{X}_{\underline{s}}^{i}\right|^{2}\right] d s .
\end{aligned}
$$


Using Assumption (A.1), the second term $\sup _{s \leq t} \mathbb{E}\left[\left|\tilde{U}_{s}^{i}-\tilde{U}_{\underline{s}}^{i}\right|^{2}\right]$ becomes

$$
\begin{aligned}
\sup _{s \leq t} \mathbb{E}\left[\left|\tilde{U}_{s}^{i}-\tilde{U}_{\underline{s}}^{i}\right|^{2}\right] \leq & 3 \sup _{s \leq t}\left\{\mathbb{E}\left[\left|\int_{\underline{s}}^{s} b\left(\tilde{X}_{\underline{r}^{-}}^{i}\right) d r\right|^{2}+\left|\int_{\underline{s}}^{s} \sigma\left(\tilde{X}_{\underline{r}^{-}}^{i}\right) d B_{r}^{i}\right|^{2}+\left|\int_{\underline{s}}^{s} \int_{E} F\left(\tilde{X}_{\underline{r}^{-}}^{i}, z\right) \tilde{N}^{i}(d r, d z)\right|^{2}\right]\right\} \\
\leq & 3 \sup _{s \leq t}\left\{\mathbb{E}\left[\left|b\left(\tilde{X}_{\underline{s}}^{i}\right)\right|^{2}|s-\underline{s}|^{2}+\left|\sigma\left(\tilde{X}_{\underline{s}}^{i}\right)\right|^{2}\left|B_{s}^{i}-B_{\underline{s}}^{i}\right|^{2}+\int_{\underline{s}}^{s} \int_{E}\left|F\left(\tilde{X}_{\underline{r}^{-}}^{i}, z\right)\right|^{2} \lambda(d z) d r\right]\right\} \\
\leq & 3 \sup _{s \leq t}\left\{\mathbb{E}\left[\left|b\left(\tilde{X}_{\underline{s}}^{i}\right)\right|^{2}|s-\underline{s}|^{2}+\left|\sigma\left(\tilde{X}_{\underline{s}}^{i}\right)\right|^{2}\left|B_{s}^{i}-B_{\underline{s}}^{i}\right|^{2}+C \int_{\underline{s}}^{s}\left(1+\left|\tilde{X}_{\underline{r}^{-}}^{i}\right|^{2}\right) d r\right]\right\} \\
\leq & 3 \sup _{s \leq t}\left\{\left(\frac{T}{n}\right)^{2} \mathbb{E}\left[\left|\sup _{\underline{s} \leq r \leq s} b\left(\tilde{X}_{\underline{r}^{-}}^{i}\right)\right|^{2}\right]+\mathbb{E}\left[\left|B_{s}^{i}-B_{\underline{s}}^{i}\right|^{2}\right] \mathbb{E}\left[\mid \sigma\left(\left.\tilde{X}_{\underline{s}}^{i}\right|^{2}\right]\right.\right. \\
& \left.+C\left(\frac{T}{n}\right) \mathbb{E}\left[\sup _{\underline{s} \leq r \leq s}\left(1+\left|\tilde{X}_{r}^{i}\right|^{2}\right)\right]\right\} \\
\leq & C_{1}\left(\frac{T}{n}\right)^{2} \mathbb{E}\left[\sup _{s \leq T}\left|b\left(\tilde{X}_{s}^{i}\right)\right|^{2}\right]+C_{2} \sup _{s \leq t} \mathbb{E}\left[\left|B_{s}^{i}-B_{\underline{s}}^{i}\right|^{2}\right] \mathbb{E}\left[\sup _{s \leq T} \mid \sigma\left(\left.\tilde{X}_{s}^{i}\right|^{2}\right]\right. \\
& +C_{3}\left(\frac{T}{n}\right) \mathbb{E}\left[\sup _{s \leq T}\left(1+\left|\tilde{X}_{s}^{i}\right|^{2}\right)\right] \\
\leq & C_{1}\left(\frac{T}{n}\right)^{2}\left(1+\mathbb{E}\left[\sup _{s \leq T}\left|\tilde{X}_{s}^{i}\right|^{2}\right]\right)+C_{2} \sup _{s \leq t} \mathbb{E}\left[\left|B_{s}^{i}-B_{\underline{s}}^{i}\right|^{2}\right]\left(1+\mathbb{E}\left[\sup _{s \leq T}\left|\tilde{X}_{s}^{i}\right|^{2}\right]\right) \\
& +C_{3}\left(\frac{T}{n}\right)\left(1+\mathbb{E}\left[\sup _{s \leq T}\left|\tilde{X}_{s}^{i}\right|^{2}\right]\right),
\end{aligned}
$$

and from Proposition 1, we get

$$
\sup _{s \leq t} \mathbb{E}\left[\left|\tilde{U}_{s}^{i}-\tilde{U}_{\underline{s}}^{i}\right|^{2}\right] \leq C_{1}\left(\frac{T}{n}\right)+C_{2} \sup _{s \leq t} \mathbb{E}\left[\left|B_{s}^{i}-B_{\underline{s}}^{i}\right|^{2}\right] .
$$

Then, by using BDG inequality, we obtain

$$
\sup _{s \leq t} \mathbb{E}\left[\left|B_{s}^{i}-B_{\underline{s}}^{i}\right|^{2}\right]=\sup _{s \leq t} \mathbb{E}\left[\left(\int_{\underline{s}}^{s} d B_{u}^{i}\right)^{2}\right] \leq \sup _{s \leq t}|s-\underline{s}| \leq \frac{T}{n} .
$$

Therefore, we conclude

$$
\begin{aligned}
\sup _{s \leq t} \mathbb{E}\left[\left|\tilde{U}_{s}^{i}-\tilde{U}_{\underline{s}}^{i}\right|^{2}\right] & \leq C_{1} n^{-1}+C_{2} n^{-1} \\
& \leq C n^{-1}
\end{aligned}
$$

from which we derive the inequality

$$
\mathbb{E}\left[\sup _{s \leq t}\left|G_{0}\left(\mu_{s}^{N}\right)-G_{0}\left(\tilde{\mu}_{\underline{s}}^{N}\right)\right|^{2}\right] \leq C\left\{n^{-1}+N^{-1 / 2}+\int_{0}^{t} \mathbb{E}\left[\left|X_{s}^{i}-\tilde{X}_{\underline{s}}^{i}\right|^{2}\right] d s\right\},
$$

and taking into account (4.2) we get

$$
\mathbb{E}\left[\sup _{s \leq t}\left|X_{s}^{i}-\tilde{X}_{s}^{i}\right|^{2}\right] \leq C\left\{n^{-1}+N^{-1 / 2}+\int_{0}^{t} \mathbb{E}\left[\left|X_{s}^{i}-\tilde{X}_{\underline{s}}^{i}\right|^{2}\right] d s\right\} .
$$


Since

$$
\begin{aligned}
\mathbb{E}\left[\left|X_{s}^{i}-\tilde{X}_{\underline{s}}^{i}\right|^{2}\right] & \leq 2 \mathbb{E}\left[\left|X_{s}^{i}-\tilde{X}_{s}^{i}\right|^{2}\right]+2 \mathbb{E}\left[\left|\tilde{X}_{s}^{i}-\tilde{X}_{\underline{s}}^{i}\right|^{2}\right] \\
& =2 \mathbb{E}\left[\left|X_{s}^{i}-\tilde{X}_{s}^{i}\right|^{2}\right]+2 \mathbb{E}\left[\left|\tilde{U}_{s}^{i}-\tilde{U}_{\underline{s}}^{i}\right|^{2}\right]
\end{aligned}
$$

it follows from (4.3) and (4.5) that

$$
\mathbb{E}\left[\sup _{s \leq t}\left|X_{s}^{i}-\tilde{X}_{s}^{i}\right|^{2}\right] \leq C\left\{n^{-1}+N^{-1 / 2}+\int_{0}^{t} \mathbb{E}\left[\left|X_{s}^{i}-\tilde{X}_{s}^{i}\right|^{2}\right] d s\right\}
$$

and finally, we conclude the proof of (i) with Gronwall's Lemma.

Proof of (ii). Following the proof of (ii) in Theorem 2, we obtain

$$
\begin{aligned}
& \mathbb{E}\left[\sup _{s \leq t}\left|\int h\left(\bar{G}_{0}\left(\mu_{s}^{i}\right)+\cdot\right)\left(d \mu_{s}^{N}-d \mu_{s}^{i}\right)\right|^{2}\right] \leq C N^{-1}, \\
& \mathbb{E}\left[\sup _{s \leq t}\left|\int h\left(\bar{G}_{0}\left(\tilde{\mu}_{\underline{s}}^{i}\right)+\cdot\right)\left(d \tilde{\mu}_{\underline{s}}^{N}-d \tilde{\mu}_{\underline{s}}^{i}\right)\right|^{2}\right] \leq C N^{-1} .
\end{aligned}
$$

By using the same strategy as the one applied in the proof of (i) in Theorem 4, the result follows easily:

$$
\mathbb{E}\left[\sup _{s \leq t}\left|X_{s}^{i}-\tilde{X}_{s}^{i}\right|^{2}\right] \leq C\left(n^{-1}+N^{-1}\right)
$$

Theorem 3. Let $T>0, N$ and $n$ be two non-negative integers and assumptions (A.1), (A.2) and (A.3) hold.

(i) There exists a constant $C$, depending on $T, b, \sigma, F, h$ and $X_{0}$ but independent of $N$, such that: for all $i=1, \ldots, N$,

$$
\mathbb{E}\left[\sup _{t \leq T}\left|\bar{X}_{t}^{i}-\tilde{X}_{t}^{i}\right|^{2}\right] \leq C\left(n^{-1}+N^{-1 / 2}\right) .
$$

(ii) If in addition (A.4) holds, there exists a positive constant $C$, depending on $T, b, \sigma, F, h$ and $X_{0}$ but independent of $N$, such that: for all $i=1, \ldots, N$,

$$
\mathbb{E}\left[\sup _{t \leq T}\left|\bar{X}_{t}^{i}-\tilde{X}_{t}^{i}\right|^{2}\right] \leq C\left(n^{-1}+N^{-1}\right) .
$$

Proof. The proof is straightforward writing

$$
\left|\bar{X}_{t}^{i}-\tilde{X}_{t}^{i}\right| \leq\left|\bar{X}_{t}^{i}-X_{t}^{i}\right|+\left|X_{t}^{i}-\tilde{X}_{t}^{i}\right|,
$$

and using Theorem 2 and Proposition 4. 


\section{NumericAl EXAMPLES.}

In this section, let us study on $[0, T]$ the following sort of processes:

$$
\left\{\begin{array}{l}
X_{t}=X_{0}-\int_{0}^{t}\left(\beta_{s}+a_{s} X_{s^{-}}\right) d s+\int_{0}^{t}\left(\sigma_{s}+\gamma_{s} X_{s^{-}}\right) d B_{s}+\int_{0}^{t} \int_{E} c(z)\left(\eta_{s}+\theta_{s} X_{s^{-}}\right) \tilde{N}(d s, d z)+K_{t}, \quad t \geq 0, \\
\mathbb{E}\left[h\left(X_{t}\right)\right] \geq 0, \quad \int_{0}^{t} \mathbb{E}\left[h\left(X_{s}\right)\right] d K_{s}=0, \quad t \geq 0,
\end{array}\right.
$$

where $\left(\beta_{t}\right)_{t \geq 0},\left(a_{t}\right)_{t \geq 0},\left(\sigma_{t}\right)_{t \geq 0},\left(\gamma_{t}\right)_{t \geq 0},\left(\eta_{t}\right)_{t \geq 0}$ and $\left(\theta_{t}\right)_{t \geq 0}$ are bounded adapted processes. This sort of processes is chosen to make some explicit computations which allow the illustration of the algorithm. Different diffusions and functions $h$ are considered in order to illustrate our results.

Linear constraint. Firstly, we consider the cases where $h: \mathbb{R} \ni x \longmapsto x-p \in \mathbb{R}$.

Case (i) Drifted Brownian motion and compensated Poisson process: $\beta_{t}=\beta>0, a_{t}=\gamma_{t}=\theta_{t}=$ $0, \sigma_{t}=\sigma>0, \eta_{t}=\eta>0, X_{0}=x_{0} \geq p, c(z)=z$ and

$$
f(z)=\frac{1}{\sqrt{2 \pi z}} \exp \left(-\frac{(\ln z)^{2}}{2}\right) \mathbf{1}_{\{\mathbf{0}<\mathbf{z}\}} .
$$

We have

$$
K_{t}=\left(p+\beta t-x_{0}\right)^{+}
$$

and

$$
X_{t}=X_{0}-(\beta+\lambda \sqrt{e}) t+\sigma B_{t}+\sum_{i=0}^{N_{t}} \eta \xi_{i}+K_{t},
$$

where $N_{t} \sim \mathcal{P}(\lambda t)$ and $\xi_{i} \sim \operatorname{lognormal}(0,1)$.

Case (ii) Black and Scholes process: $\beta_{t}=\sigma_{t}=\eta_{t}=0, a_{t}=a>0, \gamma_{t}=\gamma>0, \theta_{t}=\theta>0$, $c(z)=\delta_{1}(z)$. Then

$K_{t}=a p\left(t-t^{*}\right) 1_{t \geq t^{*}}$, where $t^{*}=\frac{1}{a}\left(\ln \left(x_{0}\right)-\ln (p)\right)$,

and

$$
X_{t}=Y_{t}+Y_{t} \int_{0}^{t} Y_{s}^{-1} d K_{s}
$$

where $Y$ is the process defined by:

$$
Y_{t}=X_{0} \exp \left(-\left(a+\gamma^{2} / 2+\lambda \theta\right) t+\gamma B_{t}\right)(1+\theta)^{N_{t}}
$$

Nonlinear constraint. Secondly, we illustrate the case of non-linear function $h$ :

$$
h: \mathbb{R} \ni x \longmapsto x+\alpha \sin (x)-p \in \mathbb{R}, \quad-1<\alpha<1,
$$

and we illustrate this case with

Case (iii) Ornstein Uhlenbeck process: $\beta_{t}=\beta>0, a_{t}=a>0, \gamma_{t}=\theta_{t}=0, \sigma_{t}=\sigma>0, \eta_{t}=\eta>0$, $X_{0}=x_{0}$ with $x_{0}>|\alpha|+p, c(z)=\delta_{1}(z)$. We obtain

$$
\mathrm{d} K_{t}=e^{-a t} \mathrm{~d} \sup _{s \leq t}\left(F_{s}^{-1}(0)\right)^{+},
$$


where for all $t$ in $[0, T]$,

$$
\begin{aligned}
F_{t}: \mathbb{R} \ni x \longmapsto & \left\{e^{-a t}\left(x_{0}-\beta\left(\frac{e^{a t}-1}{a}\right)+x\right)+\alpha \exp \left(-e^{-a t} \frac{\sigma^{2}}{2 a} \sinh (a t)\right)\right. \\
\times & {\left[\frac{1}{2}\left(\exp \left(\lambda t\left(e^{i \eta}-1\right)\right)+\exp \left(\lambda t\left(e^{-i \eta}-1\right)\right)\right) \sin \left(e^{-a t}\left(x_{0}-(\beta+\lambda \eta)\left(\frac{e^{-a t}-1}{a}\right)+x\right)\right)\right.} \\
& \left.+\frac{1}{2 i}\left(\exp \left(\lambda t\left(e^{i \eta}-1\right)\right)-\exp \left(\lambda t\left(e^{-i \eta}-1\right)\right)\right) \cos \left(e^{-a t}\left(x_{0}-(\beta+\lambda \eta)\left(\frac{e^{-a t}-1}{a}\right)+x\right)\right)\right] \\
& -p\}
\end{aligned}
$$

Remark 6. We choose these examples in order to obtain an analytic form of the "true" reflecting process $K$ which can be compared numerically with its empirical approximation $\hat{K}$. Having the exact simulation of the underlying process, we can verify the efficiency of our algorithm.

5.1. Proofs of the numerical illustrations. In order to have closed, or almost closed, expression for the compensator $K$ we introduce the process $Y$ solution to the non-reflected SDE

$$
Y_{t}=X_{0}-\int_{0}^{t}\left(\beta_{s}+a_{s} Y_{s^{-}}\right) d s+\int_{0}^{t}\left(\sigma_{s}+\gamma_{s} Y_{s^{-}}\right) d B_{s}+\int_{0}^{t} \int_{E} c(z)\left(\eta_{s}+\theta_{s} Y_{s^{-}}\right) \tilde{N}(d s, d z) .
$$

By letting $A_{s}=\int_{0}^{t} a_{s} d s$ and applying Itô's formula on $e^{A_{t}} X_{t}$ and $e^{A_{t}} Y_{t}$, we get

$$
\begin{aligned}
e^{A_{t}} X_{t}= & X_{0}+\int_{0}^{t} e^{A_{s}} X_{s} a_{s} d s+\int_{0}^{t} e^{A_{s}}\left(-\beta_{s}-a_{s} X_{s^{-}}\right) d s+\int_{0}^{t} e^{A_{s}}\left(\sigma_{s}+\gamma_{s} X_{s^{-}}\right) d B_{s} \\
& +\int_{0}^{t} \int_{E} e^{A_{s}} c(z)\left(\eta_{s}+\theta_{s} X_{s^{-}}\right) \tilde{N}(d s, d z)+\int_{0}^{t} e^{A_{s}} d K_{s} \\
= & X_{0}-\int_{0}^{t} e^{A_{s}} \beta_{s} d s+\int_{0}^{t} e^{A_{s}}\left(\sigma_{s}+\gamma_{s} X_{s^{-}}\right) d B_{s}+\int_{0}^{t} \int_{E} e^{A_{s}} c(z)\left(\eta_{s}+\theta_{s} X_{s^{-}}\right) \tilde{N}(d s, d z)+\int_{0}^{t} e^{A_{s}} d K_{s} .
\end{aligned}
$$

In the same way,

$$
e^{A_{t}} Y_{t}=X_{0}-\int_{0}^{t} e^{A_{s}} \beta_{s} d s+\int_{0}^{t} e^{A_{s}}\left(\sigma_{s}+\gamma_{s} Y_{s^{-}}\right) d B_{s}+\int_{0}^{t} \int_{E} e^{A_{s}} c(z)\left(\eta_{s}+\theta_{s} Y_{s^{-}}\right) \tilde{N}(d s, d z),
$$

and so

$$
X_{t}=Y_{t}+e^{-A_{t}} \int_{0}^{t} e^{A_{s}} d K_{s}+e^{-A_{t}} \int_{0}^{t} e^{A_{s}} \gamma_{s}\left(X_{s^{-}}+Y_{s^{-}}\right) d B_{s}+e^{-A_{t}} \int_{0}^{t} \int_{E} e^{A_{s}} c(z) \theta_{s}\left(X_{s^{-}}+Y_{s^{-}}\right) \tilde{N}(d s, d z)
$$

Remark 7. In all cases, we have $a_{t}=$ a i.e. $A_{t}=$ at, so we get

$$
\begin{aligned}
\mathbb{E}\left[Y_{t}\right] & =\mathbb{E}\left[e^{-a t}\left(x_{0}-\int_{0}^{t} e^{a s} \beta d s+\int_{0}^{t} e^{a s}\left(\sigma_{s}+\gamma_{s} Y_{s^{-}}\right) d B_{s}+\int_{0}^{t} \int_{E} e^{a s} c(z)\left(\eta_{s}+\theta_{s} Y_{s^{-}}\right) \tilde{N}(d s, d z)\right)\right] \\
& =e^{-a t}\left(x_{0}-\int_{0}^{t} e^{a s} \beta d s\right) \\
& =e^{-a t}\left(x_{0}-\beta\left(\frac{e^{a t}-1}{a}\right)\right) .
\end{aligned}
$$

Proof of assertions (i). From Proposition 3 and Remark 7, we have

$$
\begin{aligned}
& k_{t}=\beta \mathbf{1}_{\mathbb{E}\left(\mathbf{X}_{\mathbf{t}}\right)=\mathbf{p}} \\
& =\beta \mathbf{1}_{\mathbb{E}\left(\mathbf{Y}_{\mathbf{t}}\right)+\mathbf{K}_{\mathbf{t}}-\mathbf{p}=\mathbf{0}} \\
& =\beta \mathbf{1}_{\mathbf{x}_{\mathbf{0}}-\beta \mathbf{t}+\mathbf{K}_{\mathbf{t}}-\mathbf{p}=\mathbf{0}}
\end{aligned}
$$


so, we obtain that

$$
\begin{aligned}
K_{t} & =\int_{0}^{t} k_{s} d s \\
& =\int_{0}^{t} \beta \mathbf{1}_{\mathbf{K}_{\mathbf{s}}=\mathbf{p}+\beta \mathbf{s}-\mathbf{x}_{\mathbf{0}}} \mathbf{d s},
\end{aligned}
$$

and as $K_{t} \geq 0$, we conclude that

$$
K_{t}=\left(p+\beta t-x_{0}\right)^{+}
$$

Next, we have

$$
f(z)=\frac{1}{\sqrt{2 \pi z}} \exp \left(-\frac{(\ln z)^{2}}{2}\right)
$$

the density function of a lognormal random variable, so we can obtain

$$
\int_{E} \eta z \lambda(d z)=\lambda \eta \int_{E} z f(z) d z=\lambda \eta \mathbb{E}(\xi)
$$

where $\xi \sim \operatorname{lognormal}(0,1)$, and we conclude that

$$
\int_{E} \eta z \lambda(d z)=\lambda \eta \sqrt{e}
$$

Finally, we deduce the exact solution

$$
X_{t}=X_{0}-(\beta+\lambda \sqrt{e}) t+\sigma B_{t}+\sum_{i=0}^{N_{t}} \eta \xi_{i}+K_{t},
$$

where $N_{t} \sim \mathcal{P}(\lambda t)$ and $\xi_{i} \sim$ lognormal $(0,1)$.

Proof of assertions (ii). In this case, and using the same Proposition and Remark, we have

$$
k_{t}=\left(\mathbb{E}\left(-a X_{t}\right)\right)^{-} \mathbf{1}_{\mathbb{E}\left(\mathbf{X}_{\mathbf{t}}\right)=\mathbf{p}},
$$

which

$$
\begin{aligned}
\mathbb{E}\left(X_{t}\right)=p & \Longleftrightarrow \mathbb{E}\left(Y_{t}\right)-p+e^{-a t} \int_{0}^{t} e^{a s} d K_{s}=0 \\
& \Longleftrightarrow-x_{0} e^{-a t}+p=e^{-a t} \int_{0}^{t} e^{a s} d K_{s} \\
& \Longleftrightarrow K_{s}=a p,
\end{aligned}
$$

and

$$
\begin{aligned}
K_{t} \geq 0 & \Longleftrightarrow-x_{0} e^{-a t}+p \geq 0 \\
& \Longleftrightarrow e^{-a t} \leq \frac{p}{x_{0}} \\
& \Longleftrightarrow t \geq \frac{1}{a}\left(\ln \left(x_{0}\right)-\ln (p)\right):=t^{*} .
\end{aligned}
$$

So, we conclude that $K_{t}=a p\left(t-t^{*}\right) 1_{t \geq t^{*}}$, where $t^{*}=\frac{1}{a}\left(\ln \left(x_{0}\right)-\ln (p)\right)$. Next, by the definition of the process $Y_{t}$ :

$$
d Y_{t}=-a Y_{t^{-}} d t+\gamma Y_{t^{-}} d B_{t}+\theta Y_{t^{-}} d \tilde{N}_{t},
$$

we have

$$
Y_{t}=X_{0} \exp \left(-\left(a+\gamma^{2} / 2+\lambda \theta\right) t+\gamma B_{t}\right)(1+\theta)^{N_{t}}
$$


Thanks to Itô's formula we get

$$
\begin{aligned}
d\left(\frac{1}{Y_{t}}\right) & =-\frac{1}{Y_{t}^{2}} d Y_{t}+\frac{1}{2}\left(\frac{2}{Y_{t}^{3}}\right) \gamma^{2} Y_{t}^{2} d t+d \sum_{s \leq t}\left(\frac{1}{Y_{s^{-}}+\Delta Y_{s}}-\frac{1}{Y_{s^{-}}}+\frac{1}{Y_{s^{-}}^{2}} \Delta Y_{s}\right) \\
& =\frac{a}{Y_{t}} d t-\frac{\gamma}{Y_{t}} d B_{t}-\frac{\theta}{Y_{t^{-}}} d \tilde{N}_{t}+\frac{\gamma^{2}}{Y_{t}} d t+d \sum_{s \leq t}\left(\frac{1}{(1+\theta) Y_{s^{-}}}-\frac{1}{Y_{s^{-}}}+\frac{\theta}{Y_{s^{-}}}\right)
\end{aligned}
$$

and so

$$
\begin{aligned}
d Y_{t}^{-1} & =\left(a+\gamma^{2}\right) Y_{t}^{-1} d t-\gamma Y_{t}^{-1} d B_{t}-\theta Y_{t^{-}}^{-1} d \tilde{N}_{t}+\left(\frac{\theta^{2}}{1+\theta}\right) d \sum_{s \leq t} Y_{s^{-}}^{-1} \\
& =\left(a+\gamma^{2}+\frac{\lambda \theta^{2}}{1+\theta}\right) Y_{t}^{-1} d t-\gamma Y_{t}^{-1} d B_{t}-\left(\frac{\theta}{1+\theta}\right) Y_{t^{-}}^{-1} d \tilde{N}_{t} .
\end{aligned}
$$

Then, using integration by parts formula, we obtain

$$
\begin{aligned}
d\left(X_{t} Y_{t}^{-1}\right)= & X_{t^{-}} d Y_{t}^{-1}+Y_{t^{-}}^{-1} d X_{t}+d\left[X, Y^{-1}\right]_{t} \\
= & \left(a+\gamma^{2}\right) X_{t} Y_{t}^{-1} d t-\gamma X_{t} Y_{t}^{-1} d B_{t}-\theta X_{t^{-}} Y_{t^{-}}^{-1} d \tilde{N}_{t}+\left(\frac{\theta^{2}}{1+\theta}\right) d \sum_{s \leq t} X_{s^{-}} Y_{s^{-}}^{-1} \\
& -a X_{t} Y_{t}^{-1} d t+\gamma X_{t} Y_{t}^{-1} d B_{t}+\theta X_{t^{-}} Y_{t^{-}}^{-1} d \tilde{N}_{t}+Y_{t}^{-1} d K_{t} \\
& -\gamma^{2} X_{t} Y_{t}^{-1} d t-\left(\frac{\theta^{2}}{1+\theta}\right) d \sum_{s \leq t} X_{s^{-}} Y_{s^{-}}^{-1} \\
= & Y_{t}^{-1} d K_{t} .
\end{aligned}
$$

Finally, we deduce that

$$
X_{t}=Y_{t}+Y_{t} \int_{0}^{t} Y_{s}^{-1} d K_{s}
$$

Proof of assertions (iii). In that case, we have

$$
\begin{aligned}
Y_{t} & =e^{-a t}\left(x_{0}-\beta\left(\frac{e^{a t}-1}{a}\right)\right)+\sigma_{s} e^{-a t} \int_{0}^{t} e^{a s} d B_{s}+e^{-a t} \int_{0}^{t} \eta_{s} e^{a s} d \tilde{N}_{s} \\
& =e^{-a t}\left(x_{0}-(\beta+\lambda \eta)\left(\frac{e^{a t}-1}{a}\right)\right)+\sigma_{s} e^{-a t} \int_{0}^{t} e^{a s} d B_{s}+e^{-a t} \int_{0}^{t} \eta_{s} e^{a s} d N_{s} \\
& :=f_{t}+G_{t}+F_{t},
\end{aligned}
$$

and

Hence

$$
X_{t}=Y_{t}+e^{-a t} \bar{K}_{t}, \quad \bar{K}_{t}=\int_{0}^{t} e^{a s} d K_{s} .
$$

$$
\begin{aligned}
h\left(X_{t}\right)= & Y_{t}+e^{-a t} \bar{K}_{t}+\alpha \sin \left(Y_{t}+e^{-a t} \bar{K}_{t}\right)-p \\
= & Y_{t}+e^{-a t} \bar{K}_{t}+\alpha\left(\sin \left(Y_{t}\right) \cos \left(e^{-a t} \bar{K}_{t}\right)+\cos \left(Y_{t}\right) \sin \left(e^{-a t} \bar{K}_{t}\right)\right)-p \\
= & Y_{t}+e^{-a t} \bar{K}_{t}+\alpha\left[\operatorname { c o s } ( e ^ { - a t } \overline { K } _ { t } ) \left\{\sin \left(f_{t}\right) \cos \left(G_{t}\right) \cos \left(F_{t}\right)+\cos \left(f_{t}\right) \sin \left(G_{t}\right) \cos \left(F_{t}\right)\right.\right. \\
& \left.+\cos \left(f_{t}\right) \cos \left(G_{t}\right) \sin \left(F_{t}\right)-\sin \left(f_{t}\right) \sin \left(G_{t}\right) \sin \left(F_{t}\right)\right\}+\sin \left(e^{-a t} \bar{K}_{t}\right)\left\{\cos \left(f_{t}\right) \cos \left(G_{t}\right) \cos \left(F_{t}\right)\right. \\
& \left.\left.-\sin \left(f_{t}\right) \sin \left(G_{t}\right) \sin \left(F_{t}\right)-\sin \left(f_{t}\right) \cos \left(G_{t}\right) \sin \left(F_{t}\right)-\cos \left(f_{t}\right) \sin \left(G_{t}\right) \sin \left(F_{t}\right)\right\}\right]-p .
\end{aligned}
$$



On one side, since $G_{t}$ is a centered gaussian random variable with variance $V=\sigma^{2} \frac{1-e^{-2 a t}}{2 a}=\sigma^{2} e^{-a t} \frac{\sinh (a t)}{a}$,
we obtain that

$$
\begin{gathered}
\mathbb{E}\left[e^{i G_{t}}\right]=e^{-V / 2}, \\
\mathbb{E}\left[\sin \left(G_{t}\right)\right]=\mathbb{E}\left[\frac{e^{i G_{t}}-e^{-i G_{t}}}{2 i}\right]=0,
\end{gathered}
$$

and

$$
\mathbb{E}\left[\cos \left(G_{t}\right)\right]=\mathbb{E}\left[\frac{e^{i G_{t}}+e^{-i G_{t}}}{2}\right]=\mathbb{E}\left(e^{i G_{t}}\right)=\exp \left(-e^{-a t} \frac{\sigma^{2}}{2 a} \sinh (a t)\right)=: g(t) .
$$

On the other side,

by taking ' $a$ ' small, we get

$$
\mathbb{E}\left[e^{i F_{t}}\right]=\mathbb{E}\left[\exp \left(i \eta e^{-a t} \int_{0}^{t} e^{a s} d N_{s}\right)\right],
$$

and so

$$
\begin{aligned}
\mathbb{E}\left[e^{i F_{t}}\right] & \approx \mathbb{E}\left[\exp \left(i \eta \int_{0}^{t} d N_{s}\right)\right] \\
& \approx \mathbb{E}\left[\exp \left(i \eta N_{t}\right)\right] \\
& \approx \exp \left(\lambda t\left(e^{i \eta}-1\right)\right),
\end{aligned}
$$

$$
\begin{aligned}
& \mathbb{E}\left[\sin \left(F_{t}\right)\right] \approx \frac{\exp \left(\lambda t\left(e^{i \eta}-1\right)\right)-\exp \left(\lambda t\left(e^{-i \eta}-1\right)\right)}{2 i}=: m(t), \\
& \mathbb{E}\left[\cos \left(F_{t}\right)\right] \approx \frac{\exp \left(\lambda t\left(e^{i \eta}-1\right)\right)+\exp \left(\lambda t\left(e^{-i \eta}-1\right)\right)}{2}=: n(t) .
\end{aligned}
$$

Using Remark 7, we conclude that, for small ' $a$ ',

$$
\begin{aligned}
\mathbb{E}\left[h\left(X_{t}\right)\right] & \approx \mathbb{E}\left[Y_{t}\right]+e^{-a t} \bar{K}_{t}+\alpha\left(g(t) m(t) \cos \left(f_{t}+e^{-a t} \bar{K}_{t}\right)+g(t) n(t) \sin \left(f_{t}+e^{-a t} \bar{K}_{t}\right)\right)-p \\
& :=F_{t}\left(\bar{K}_{t}\right) .
\end{aligned}
$$

Therefore,

$$
\bar{K}_{t}=\sup _{s \leq t}\left(F_{s}^{-1}(0)\right)^{+} \quad \text { and } \quad d K_{t}=e^{-a t} d \sup _{s \leq t}\left(F_{s}^{-1}(0)\right)^{+}
$$

5.2. Illustrations. This computation works as follows. Let $0=T_{0}<T_{1}<\cdots<T_{n}=T$ be a subdivision of $[0, T]$ of step size $T / n, n$ being a positive integer, let $X$ be the unique solution of the MRSDE (5.1) and let, for a given $i,\left(\tilde{X}_{T_{k}}^{i}\right)_{0 \leq k \leq n}$ be its numerical approximation given by Algorithm 1. For a given integer $L$, we draw $\left(\bar{X}^{l}\right)_{0 \leq l \leq L}$ and $\left(\tilde{X}^{i, l}\right)_{0 \leq l \leq L}, L$ independent copies of $X$ and $\tilde{X}^{i}$. Then, we approximate the $\mathbb{L}^{2}$-error of Theorem 3 by:

$$
\hat{E}=\frac{1}{L} \sum_{l=1}^{L} \max _{0 \leq k \leq n}\left|\bar{X}_{T_{k}}^{l}-\tilde{X}_{T_{k}}^{i, l}\right|^{2}
$$

Figure 1 illustrates the evolution in time of the true $K$ (full line) and the estimated $K$ (dotted line for particle method) (dashed line for density method) in case (i). It is confirmed that the approximation of $K$ is almost the same as the exact solution. The evolution of $\log (\hat{E})$ w.r.t. $\log (N)$ is depicted in Figure 2. It can be seen that the slope is equal to 0.9, which is consistent with the statement of Theorem 3. 


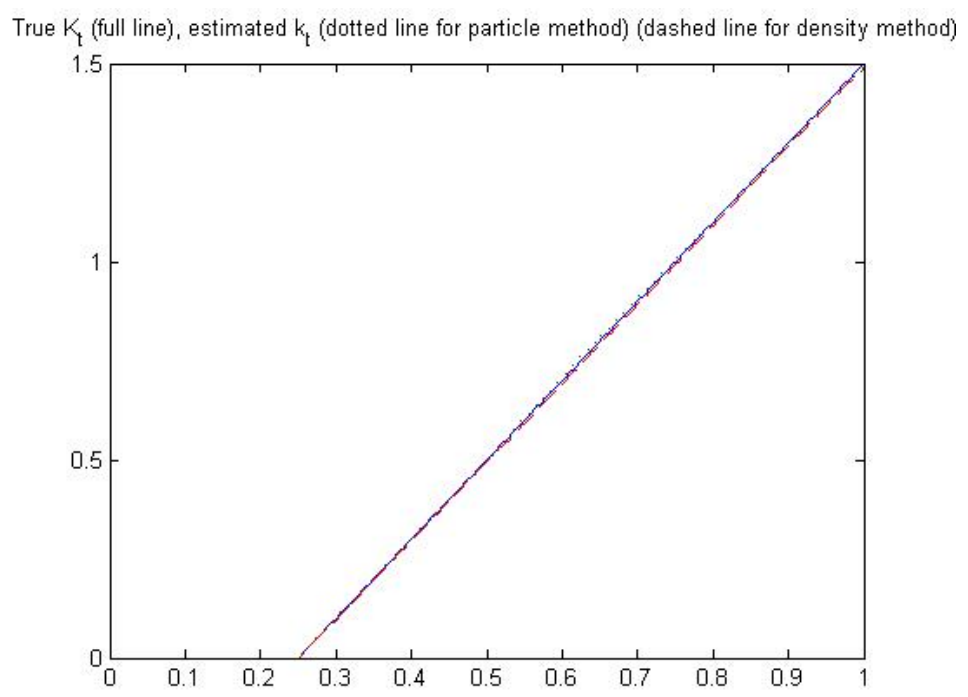

Figure 1. Case (i). $n=500, N=100000, T=1, \beta=2, \sigma=1, \lambda=5, x_{0}=$ $1, p=1 / 2$.

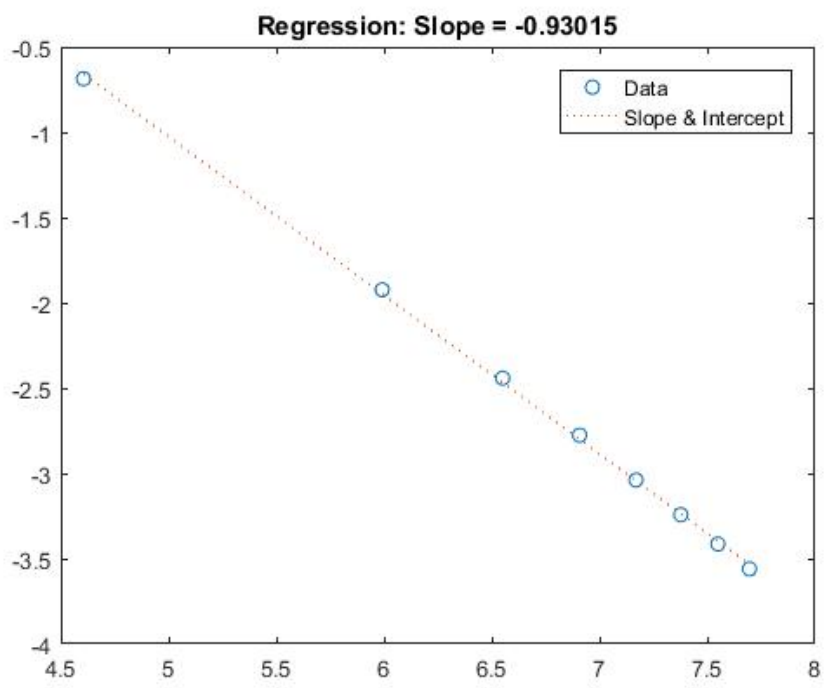

Figure 2. Case (i). Regression of $\log (\hat{E})$ w.r.t. $\log (N)$. Data: $\hat{E}$ when $N$ varies from 100 to 2200 with step size 300. Parameters: $n=100, T=1, \beta=2, \sigma=1$, $\lambda=5, x_{0}=1, p=1 / 2, L=1000$.

Figure 3 illustrates the evolution in time of the true $K$ (full line) and the estimated $K$ (dotted line for particle method) (dashed line for density method) in case (ii). As in the previous example, the approximation of $K$ is almost the same as the exact solution. The evolution of $\log (\hat{E})$ w.r.t. $\log (N)$ is depicted in Figure 4. It can be seen that the slope is equal to 0.9, which is consistent with the statement of Theorem 3 . 


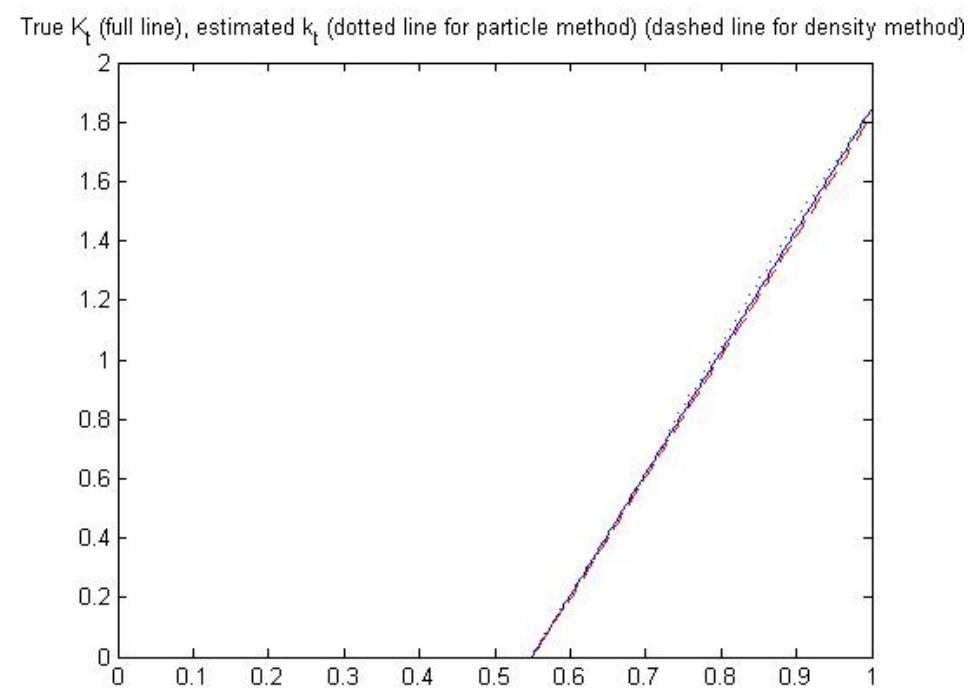

Figure 3. Case (ii). Parameters: $n=500, N=10000, T=1, \beta=0, a=3$, $\gamma=1, \eta=1, \lambda=2, x_{0}=4, p=1$.

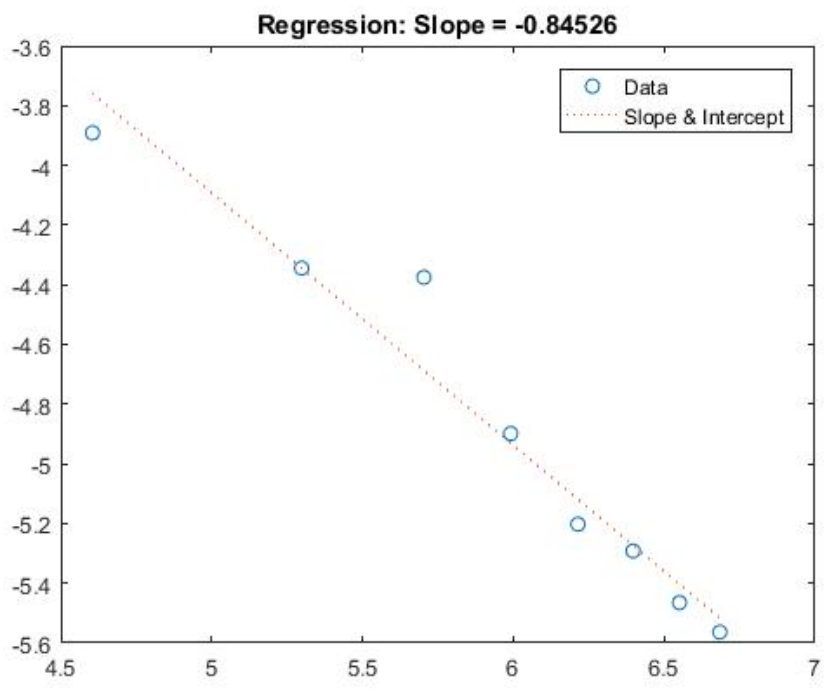

Figure 4. Case (ii). Regression of $\log (\hat{E})$ w.r.t. $\log (N)$. Data: $\hat{E}$ when $N$ varies from 100 to 800 with step size 100. Parameters: $n=1000, T=1, \beta=0, a=3$, $\gamma=1, \eta=1, \lambda=2, x_{0}=4, p=1, L=1000$.

Figure 5 illustrates the evolution in time of the true $K$ (full line) and the estimated $K$ (dotted line for particle method) (dashed line for density method) in case (iii). Moreover, we notice that the approximation of $K$ with particle method is closer to the exact $K$ than the one with density method. 


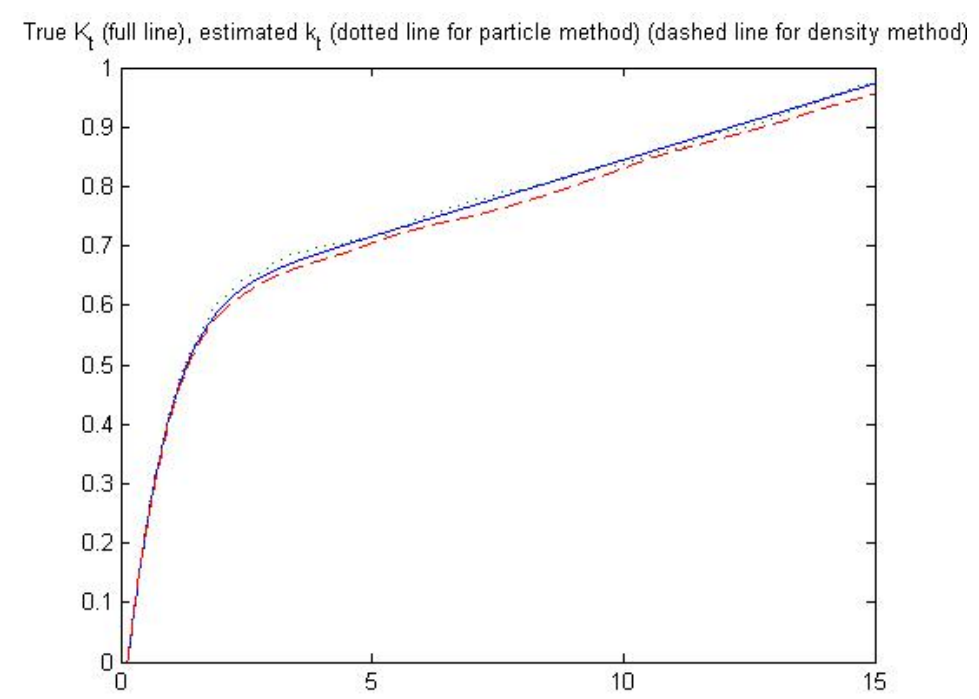

Figure 5. Case (iii). Parameters: $n=1000, N=100000, T=15, \beta=10^{-2}$, $\sigma=1, p=\pi / 2, \alpha=0.9, a=10^{-2}, x_{0}$ is the unique solution of $x+\alpha \sin (x)-p=0$ plus $10^{-1}$.

\section{Appendix A. Appendices}

A.1. Proof of Lemma 4. Let $s$ and $t$ in $[0, T]$ be such that $s \leq t$.

Firstly, we suppose that $\varphi$ is a continuous function with compact support. In this case, there exists a sequence of Lipschitz continuous functions $\varphi_{n}$ with compact support which converges uniformly to $\varphi$. Therefore, by using Proposition 2, we get

$$
\begin{aligned}
\left|\mathbb{E}\left[\varphi\left(X_{t}\right)\right]-\mathbb{E}\left[\varphi\left(X_{s}\right)\right]\right| & \leq\left|\mathbb{E}\left[\varphi\left(X_{t}\right)\right]-\mathbb{E}\left[\varphi_{n}\left(X_{t}\right)\right]\right|+\left|\mathbb{E}\left[\varphi_{n}\left(X_{t}\right)\right]-\mathbb{E}\left[\varphi_{n}\left(X_{s}\right)\right]\right|+\left|\mathbb{E}\left[\varphi_{n}\left(X_{s}\right)\right]-\mathbb{E}\left[\varphi\left(X_{s}\right)\right]\right| \\
& \leq \mathbb{E}\left[\left|\left(\varphi-\varphi_{n}\right)\left(X_{t}\right)\right|\right]+C_{n} \mathbb{E}\left[\left|X_{t}-X_{s}\right|\right]+\left|\mathbb{E}\left[\left(\varphi_{n}-\varphi\right)\left(X_{s}\right)\right]\right| \\
& \leq 2 \mathbb{E}\left[\left\|\varphi_{n}-\varphi\right\|_{\infty}\right]+C_{n}\left(\mathbb{E}\left[\left|X_{t}-X_{s}\right|^{2}\right]\right)^{1 / 2} \\
& \leq 2 \mathbb{E}\left[\left\|\varphi_{n}-\varphi\right\|_{\infty}\right]+C_{n}|t-s|^{1 / 2} .
\end{aligned}
$$

Thus, we obtain that

$$
\limsup _{t \rightarrow s}\left|\mathbb{E}\left[\varphi\left(X_{t}\right)\right]-\mathbb{E}\left[\varphi\left(X_{s}\right)\right]\right| \leq 2 \mathbb{E}\left[\left\|\varphi_{n}-\varphi\right\|_{\infty}\right] .
$$

This result is true for all $n \geq 1$, so we deduce that

$$
\limsup _{t \rightarrow s}\left|\mathbb{E}\left[\varphi\left(X_{t}\right)\right]-\mathbb{E}\left[\varphi\left(X_{s}\right)\right]\right|=0,
$$

then we conclude the continuity of the function $t \longmapsto \mathbb{E}\left[\varphi\left(X_{t}\right)\right]$.

Secondly, we consider the case where $\varphi$ is a continuous function such that

$$
\forall x \in \mathbb{R}, \exists C \in \mathbb{R}, \quad \varphi(x) \leq C\left(1+|x|^{p}\right) .
$$

We define a sequence of functions $\varphi_{n}$, such that for all $n \geq 1$ and $x \in \mathbb{R}$,

$$
\varphi_{n}(x)=\varphi(x) \theta_{n}(x)
$$

with

$$
\theta_{n}(x)= \begin{cases}1 & \text { if }|x| \leq n \\ 0 & \text { if }|x|>n\end{cases}
$$


Based on this definition, $\varphi_{n}$ is a continuous function with compact support. Then we get

$$
\begin{aligned}
\left|\mathbb{E}\left[\varphi\left(X_{t}\right)\right]-\mathbb{E}\left[\varphi\left(X_{s}\right)\right]\right| \leq & \left|\mathbb{E}\left[\left(\varphi-\varphi_{n}\right)\left(X_{t}\right)\left(\mathbf{1}_{\left|\mathbf{X}_{\mathbf{t}}\right| \leq \mathbf{n}}+\mathbf{1}_{\left|\mathbf{X}_{\mathbf{t}}\right|>\mathbf{n}}\right)\right]\right|+\left|\mathbb{E}\left[\varphi_{\mathbf{n}}\left(\mathbf{X}_{\mathbf{t}}\right)\right]-\mathbb{E}\left[\varphi_{\mathbf{n}}\left(\mathbf{X}_{\mathbf{s}}\right)\right]\right| \\
& +\left|\mathbb{E}\left[\left(\varphi_{n}-\varphi\right)\left(X_{s}\right)\left(\mathbf{1}_{\left|\mathbf{X}_{\mathbf{s}}\right| \leq \mathbf{n}}+\mathbf{1}_{\left|\mathbf{X}_{\mathbf{s}}\right|>\mathbf{n}}\right)\right]\right| \\
\leq & \left|\mathbb{E}\left[\left(\varphi-\varphi_{n}\right)\left(X_{t}\right) \mathbf{1}_{\left|\mathbf{X}_{\mathbf{t}}\right|>\mathbf{n}}\right]\right|+\left|\mathbb{E}\left[\varphi_{\mathbf{n}}\left(\mathbf{X}_{\mathbf{t}}\right)\right]-\mathbb{E}\left[\varphi_{\mathbf{n}}\left(\mathbf{X}_{\mathbf{s}}\right)\right]\right|+\left|\mathbb{E}\left[\left(\varphi_{\mathbf{n}}-\varphi\right)\left(\mathbf{X}_{\mathbf{s}}\right) \mathbf{1}_{\left|\mathbf{X}_{\mathbf{s}}\right|>\mathbf{n}}\right]\right| \\
\leq & 2 \mathbb{E}\left[\left|\varphi\left(X_{t}\right)\right| \mathbf{1}_{\left.\left|\mathbf{X}_{\mathbf{t}}\right|>\mathbf{n}\right]}\right]+\left|\mathbb{E}\left[\varphi_{\mathbf{n}}\left(\mathbf{X}_{\mathbf{t}}\right)\right]-\mathbb{E}\left[\varphi_{\mathbf{n}}\left(\mathbf{X}_{\mathbf{s}}\right)\right]\right|+\mathbf{2} \mathbb{E}\left[\left|\varphi\left(\mathbf{X}_{\mathbf{s}}\right)\right| \mathbf{1}_{\left|\mathbf{X}_{\mathbf{s}}\right|>\mathbf{n}}\right] \\
\leq & C \mathbb{E}\left[\left(1+\left|X_{t}\right|^{p}\right) \mathbf{1}_{\left|\mathbf{X}_{\mathbf{t}}\right|>\mathbf{n}}\right]+\left|\mathbb{E}\left[\varphi_{\mathbf{n}}\left(\mathbf{X}_{\mathbf{t}}\right)\right]-\mathbb{E}\left[\varphi_{\mathbf{n}}\left(\mathbf{X}_{\mathbf{s}}\right)\right]\right|+\mathbf{C E}\left[\left(\mathbf{1}+\left|\mathbf{X}_{\mathbf{s}}\right|^{\mathbf{p}}\right) \mathbf{1}_{\left|\mathbf{X}_{\mathbf{s}}\right|>\mathbf{n}}\right] \\
\leq & C \mathbb{E}\left[\left(1+\sup _{t \leq T}\left|X_{t}\right|^{p}\right) \mathbf{1}_{\text {sup }_{\mathbf{t} \leq \mathbf{T}}\left|\mathbf{X}_{\mathbf{t}}\right|>\mathbf{n}}\right]+\left|\mathbb{E}\left[\varphi_{\mathbf{n}}\left(\mathbf{X}_{\mathbf{t}}\right)\right]-\mathbb{E}\left[\varphi_{\mathbf{n}}\left(\mathbf{X}_{\mathbf{s}}\right)\right]\right|
\end{aligned}
$$

Thus, by using the first part of this Lemma, we obtain that

$$
\limsup _{t \rightarrow s}\left|\mathbb{E}\left[\varphi\left(X_{t}\right)\right]-\mathbb{E}\left[\varphi\left(X_{s}\right)\right]\right| \leq C \mathbb{E}\left[\left(1+\sup _{t \leq T}\left|X_{t}\right|^{p}\right) \mathbf{1}_{\text {sup } \left._{\mathbf{t} \leq \mathbf{T}}\left|\mathbf{X}_{\mathbf{t}}\right|>\mathbf{n}\right] .}\right.
$$

This result is true for all $n \geq 1$, then by using the dominated convergence theorem, we deduce that

$$
\limsup _{t \rightarrow s}\left|\mathbb{E}\left[\varphi\left(X_{t}\right)\right]-\mathbb{E}\left[\varphi\left(X_{s}\right)\right]\right|=0
$$

and we conclude the continuity of the function $t \longmapsto \mathbb{E}\left[\varphi\left(X_{t}\right)\right]$.

\section{REFERENCES}

[ADEH99] Philippe Artzner, Freddy Delbaen, Jean-Marc Eber, and David Heath. Coherent measures of risk. Math. Finance, 9(3):203-228, 1999.

[BCdRGL16] Philippe Briand, Paul-Eric Chaudru de Raynal, Arnaud Guillin, and Céline Labart. Particles systems and numerical schemes for mean reflected stochastic differential equations. arXiv:1612.06886, 2016. Submitted.

[BEH18] Philippe Briand, Romuald Elie, and Ying Hu. BSDEs with mean reflexion. Ann. Appl. Probab., 28(1):482-510, 2018.

[CD18a] R. Carmona and F. Delarue. Probabilistic Theory of Mean Field Games with Applications I. Springer, 2018.

[CD18b] R. Carmona and F. Delarue. Probabilistic Theory of Mean Field Games with Applications II. Springer, 2018.

[CM08] Stéphane Crépey and Anis Matoussi. Reflected and doubly reflected BSDEs with jumps: a priori estimates and comparison. Ann. Appl. Probab., 18(5):2041-2069, 2008.

[DL16a] R. Dumitrescu and C. Labart. Numerical approximation of doubly reflected bsdes with jumps and rcll obstacles. Journal of Math. Anal. and Appl., 442(1):206-243, 2016.

[DL16b] R. Dumitrescu and C. Labart. Reflected scheme for doubly reflected bsdes with jumps and rcll obstacles. Journal of Comput. and Appl. Math., 296:827-839, 2016.

[EHO05] E.H. Essaky, N. Harraj, and Y. Ouknine. Backward stochastic differential equation with two reflecting barriers and jumps. Stochastic Analysis and Applications, 23:921-938, 2005.

[Ess08] E.H. Essaky. Reflected backward stochastic differential equation with jumps and RCLL obstacle. Bulletin des Sciences Mathématiques, (132):690-710, 2008.

[FG15] Nicolas Fournier and Arnaud Guillin. On the rate of convergence in wasserstein distance of the empirical measure. Probab. Theory Related Fields, (162(3-4)):707--738, 2015.

[FS02] Hans Föllmer and Alexander Schied. Convex measures of risk and trading constraints. Finance Stoch., 6(4):429-447, 2002.

[HH06] S. Hamadène and M. Hassani. BSDEs with two reacting barriers driven by a Brownian motion and an independent Poisson noise and related Dynkin game. Electronic Journal of Probability, 11:121$145,2006$.

[HO03] S. Hamadène and Y. Ouknine. Reflected backward stochastic differential equation with jumps and random obstable. Elec. Journ. of Prob., 8:1-20, 2003.

[KH92] Arturo Kohatsu-Higa. Reflecting stochastic differential equations with jumps. Technical report, 1992. 
[Lep95] D. Lepingle. Euler scheme for reflected stochastic differential equations. Math. Comput. Simulation, 38:119-126, 1995.

[LL06a] J.-M. Lasry and P.-L. Lions. Jeux à champ moyen. i. le cas stationnaire. C.R. Math. Acad. Sci. Paris, 343(9):619-625, 2006.

[LL06b] J.-M. Lasry and P.-L. Lions. Jeux à champ moyen. ii. horizon fini et contrôle optimal. C.R. Math. Acad. Sci. Paris, 343(10):679-684, 2006.

[LL07a] J.-M. Lasry and P.-L. Lions. Large investor trading impacts on volatility. Ann. Inst. H. Poincaré, Anal. Non Linéaire, 24(2):311-323, 2007.

[LL07b] J.-M. Lasry and P.-L. Lions. Mean field games. Japanese Journal Math., 2:229-260, 2007.

[MR85] José-Luis Menaldi and Maurice Robin. Reflected diffusion processes with jumps. The Annals of Probability, 13(2):319-341, 1985.

[Pet95] R. Petterson. Approximations for stochastic differential equations with reflecting convex boundaries. Stoc. Proc. Appl, 59(2):295-308, 1995.

[Pet97] R. Petterson. Penalization schemes for reflecting stochastic differential equations. Bernoulli, 3(4):403-414, 1997.

[QS14] M.C. Quenez and A. Sulem. Reflected BSDEs and robust optimal stopping for dynamic risk measures with jumps. Stoc. Proc. Appl., (124(9)):3031-3054, 2014.

[Sko61] A.V. Skorokhod. Stochastic equations for diffusion processes in a bounded region. Theory Probab. Appl, 6:264-274, 1961.

[Slo94] L. Slominski. On approximation of solutions of multidimensional sdes with reflecting boundary conditions. Stoc. Proc. Appl, 50(2):197-219, 1994.

[Slo01] L. Slominski. Euler's approximations of solutions of sdes with reflecting boundary. Stoc. Proc. Appl, 92(2):317-337, 2001.

[STR98] Ludger Rüschendorf S. T. Rachev. Mass transportation problems. Vol. 1: Theory. Vol. 2: Applications. 1998.

[YS12] Hui Yu and Minghui Song. Numerical solutions of stochastic differential equations driven by poisson random measure with non-lipschitz coefficients. Journal of Applied Mathematics, 2012, 2012.

Univ. Grenoble Alpes, Univ. Savoie Mont Blanc, CnRs, lama, 73000 Chambéry, France

Email address: philippe.briand@univ-smb.fr

Univ. Grenoble Alpes, Univ. Savoie Mont Blanc, CNRS, lama, 73000 Chambéry, France

Univ. Libanaise, LaMA-Liban, P.O. Box 37, Tripoli, Liban

Email address: abir.ghannoum@univ-smb.fr

Univ. Grenoble Alpes, Univ. Savoie Mont Blanc, CNRS, lamA, 73000 Chambéry, France

Email address: celine.labart@univ-smb.fr 